\title{
Termination for Direct Sums of Left-Linear Complete Term Rewriting Systems
}

\author{
Y. TOYAMA \\ JAIST, Tatsunokuchi, Ishikawa, Japan
}

J. W. KLOP

CWI, Amsterdam, The Netherlands and Free University, Amsterdam, The Netherlands

AND

H. P. BARENDREGT

Catholic University, Nijmegen, The Netherlands

\begin{abstract}
A term rewriting system is called complete if it is confluent and terminating. We prove that completeness of TRSs is a "modular" property (meaning that it stays preserved under direct sums), provided the constituent TRSs are left-linear. Here, the direct sum $R_{0} \oplus R_{1}$ is the union of TRSs $R_{0}, R_{1}$ with disjoint signature. The proof hinges crucially upon the (non)deterministic collapsing behavior of terms from the sum TRS.

Categories and Subject Descriptors: F.4.2 [Mathematical Logic and Formal Languages]: Grammars and Other Rewriting Systems

General Terms: Theory

Additional Key Words and Phrases: Confluence, left-linearity, term rewriting systems
\end{abstract}

\section{Introduction}

An important concern in building algebraic specifications is their hierarchical or modular structure. The same holds for term rewriting systems (see Huet \& Oppen [1980], Klop [1992], or Dershowitz and Jouannaud [1990]), which can be viewed as implementations of equational algebraic specifications. Specifically, it is of obvious interest to determine which properties of term rewriting systems

J. W. Klop was partially supported by ESPRIT project 432. An Integrated Formal Approach to Industrial Software Development (METEOR).

Authors' addresses: Y. Toyama, School of Information Science, JAIST, Tatsunokuchi, Ishikawa 923-12, Japan; J. W. Klop, CWI, Kruislaan 413, 1098-SJ Amsterdam, The Netherlands, and Free University, de Boelelaan 1081, 1081 HV Amsterdam, The Netherlands; H. P. Barendregt, Faculty of Mathematics and Computer Science, Catholic University, Toernooiveld 1, 6525 ED Nijmegen, The Netherlands.

Permission to make digital/hard copy of part or all of this work for personal or classroom use is granted without fee provided that copies are not made or distributed for profit or commercial advantage, the copyright notice, the title of the publication, and its date appear, and notice is given that copying is by permission of ACM, Inc. To copy otherwise, to republish, to post on servers, or to redistribute to lists, requires prior specific permission and/or a fee.

(C) 1995 ACM 0004-5411/95/1100-1275 \$03.50 
(TRSs) have a "modular" character. We call a property modular if its validity for a TRS, hierarchically composed of some smaller TRSs, can be inferred from the validity of that property for the constituent TRSs. Naturally, the first step in such an investigation considers the most basic properties of TRSs: confluence, termination, unique normal form property, and similar fundamental properties as well as combinations thereof.

As to the modular structure of TRSs, it is again natural to consider as a start the most simple way that TRSs can be combined to form a larger TRS: namely, as a disjoint sum. This means that the alphabets of the TRSs to be combined are disjoint, and that the rewrite rules of the sum TRS are the rules of the summand TRSs together. (Without the disjointness requirement, the situation is even more complicated-for some results in this direction, see Dershowitz [1981], Toyama [1988], and Middeldorp and Toyama [1991].) A disjoint union of two TRSs $R_{0}, R_{1}$ is called in our paper, a direct sum, notation $R_{0} \oplus R_{1}$.

Another simplifying assumption that we will make is that $R_{0}, R_{1}$ are homogeneous TRSs, that is, their signature is one-sorted (as opposed to the many-sorted or heterogeneous case; for results about direct sums of heterogeneous TRSs, see Ganzinger and Giegerich [1987]).

The first result in this setting is due to Toyama [1987a], where it is proved that confluence is a modular property. That is, $R_{0} \oplus R_{1}$ is confluent $\Leftrightarrow R_{0}$ and $R_{1}$ are confluent. Here " $\Rightarrow$ " is trivial; " $\Leftarrow "$ is what we are interested in. (For a simplified proof, see Klop et al. [1994].) To appreciate the nontriviality of this fact, it may be contrasted with the fact that another fundamental property, termination, is not modular, as the following simple counterexample in Toyama [1987b] shows:

$$
\begin{aligned}
& R_{0}=\{F(0,1, x) \rightarrow F(x, x, x)\} \\
& R_{1}=\{G(x, y) \rightarrow x, G(x, y) \rightarrow y\} .
\end{aligned}
$$

It is trivial that $R_{0}$ and $R_{1}$ are terminating. However, $R_{0} \oplus R_{1}$ is not terminating, because $R_{0} \oplus R_{1}$ has the infinite reduction sequence:

$$
\begin{aligned}
& F(G(0,1), G(0,1), G(0,1)) \rightarrow F(0, G(0,1), G(0,1)) \rightarrow F(0,1, G(0,1))) \\
& \rightarrow F(G(0,1), G(0,1), G(0,1)) \rightarrow \cdots .
\end{aligned}
$$

However, this counterexample uses a non-confluent TRS $R_{1}$. A more complicated counterexample to the modularity of "termination," involving only confluent TRSs, was given by J. W. Klop and H. P. Barendregt (private communication) (for ground terms only). (For this counterexample as well as for some improved versions, holding for open terms as well, and even using TRSs that are "irreducible," see Toyama [1987b].) Rephrased, this means that the important property of "completeness" of TRSs (a TRS is complete if it is both confluent and terminating) is not modular, that is, there are complete TRSs $R_{0}, R_{1}$ such that $R_{0} \oplus R_{1}$ is not complete (in fact, not terminating; confluence of $R_{0} \oplus R_{1}$ is ensured by the theorem in Toyama [1987a]). This counterexample, however, uses non-left-linear TRSs.

The point of the present paper is that left-linearity is essential; if we restrict ourselves to left-linear TRSs, then completeness is modular. Thus, we prove: If $R_{0}, R_{1}$ are left-linear (meaning that the rewrite rules have no repeated 
variables in their left-hand sides), then $R_{0} \oplus R_{1}$ is complete iff $R_{0}, R_{1}$ are. As left-linearity is a property that can be easily checked, and many equational algebraic specifications can be given by left-linear TRSs, we feel that this result is worthwhile.

The proof, however, is rather intricate and not easily digested. A crucial element in the proof, and in general in the way that the summand TRSs interact, is how terms may "collapse" to a subterm. The problem is that this collapsing behavior may exhibit a "nondeterministic" feature, which is caused by ambiguities among the rewrite rules. We hope that the present paper is of value not only because it establishes a result that in itself is simple enough, but also because of the analysis necessary for the proof that gives a kind of structure theory for disjoint combinations of TRSs and that may be of relevance to other similar studies.

Regarding the question of modular properties in the present simple set-up, we mention the results by Rusinowitch [1987] and Middeldorp [1989a]. These papers, together, contain a complete analysis of the cases in which termination for $R_{0} \oplus R_{1}$ may be concluded from termination of $R_{0}, R_{1}$, depending on the distribution among $R_{0}, R_{1}$ of so-called collapsing and duplicating rules. Below, we will compare these results with ours.

Another useful fact is established in Middeldorp [1989b], where it is proved that the "unique normal form property" is a modular property. A survey of modularity results is in Middeldorp [1990]. For more modularity results, also pertaining to a special form of termination called simple termination, we refer to Kurihara and Kaji [1988/1990] and Kurihara and Ohuchi [1989/1990].

From now on we assume that the two TRSs $R_{0}$ and $R_{1}$ are terminating Without the assumption about the disjointness of $R_{0}$ and $R_{1}$, Bachmair anc Dershowitz [1986] have shown: If $R_{0}$ is left-linear, $R_{1}$ is right-linear, and there is no overlap between left-hand sides of $R_{0}$ and right-hand sides of $R_{1}$, then the combined system $R_{0} \cup R_{1}$ is terminating.

If $R_{0}$ and $R_{1}$ are disjoint and $R_{1}$ is noncollapsing, then nonoverlapping between left-hand sides of $R_{0}$ and right-hand sides of $R_{1}$ is trivial. Here, note that the noncollapsing property of $R_{1}$ is necessary since a variable right-hand side overlaps any left-hand side. Hence, from Bachmair and Dershowitz's theorem, it follows that if $R_{0}$ is left-linear, $R_{1}$ is right-linear and noncollapsing, then the direct sum $R_{0} \oplus R_{1}$ is terminating. However, using the disjointness of $R_{0}$ and $R_{1}$ we can strengthen this result somewhat. Indeed, it holds that if $R_{1}$ is right-linear and noncollapsing, then the direct sum $R_{0} \oplus R_{1}$ is terminating. This is a special case of Middeldorp's [1989a] theorem: If $R_{1}$ is noncollapsing and nonduplicating, then the direct sum $R_{0} \oplus R_{1}$ is terminating. Here, the properties "noncollapsing" and "nonduplicating" (with right-linearity as a special case) of TRSs are seen to be basic criteria for termination.

The point of our result is that it shows that "left-linearity and confluence" is also a basic criterion for termination of the direct sum $R_{0} \oplus R_{1}$. Indeed, in the present paper, we prove that, if $R_{0}$ and $R_{1}$ are left-linear and confluent, then the direct sum $R_{0} \oplus R_{1}$ is terminating. Rusinowitch [1987] has proved:

(A) If $R_{0}$ and $R_{1}$ are noncollapsing, then the direct sum $R_{0} \oplus R_{1}$ is terminating.

(B) If $R_{0}$ and $R_{1}$ are nonduplicating, then the direct sum $R_{0} \oplus R_{1}$ is terminating. 
So, a natural question from the observations above is whether "noncollapsing" (or "nonduplicating") and "left-linear and confluent" are interchangeable as follows:

(A') If $R_{0}$ is noncollapsing and $R_{1}$ is left-linear and confluent, then the direct sum $R_{0} \oplus R_{1}$ is terminating.

( $\left.\mathrm{B}^{\prime}\right)$ If $R_{0}$ is nonduplicating and $R_{1}$ is left-linear and confluent, then the direct sum $R_{0} \oplus R_{1}$ is terminating.

We can prove that Conjecture $\mathrm{A}^{\prime}$ holds; the proof is in Appendix $\mathrm{B}$. However, Conjecture B' does not hold because of Toyama's [1987a] counterexample.

Finally, we mention why, in this paper, we cannot use the proof method developed by Breazu-Tannen and Gallier [1989] and Okada [1989] in their results about termination of the union of the (polymorphic) typed lambda calculus $(\lambda)$ and a complete term rewriting system $R$. The reason is that their proof method is essentially analogous to the one for Conjecture $A^{\prime}$ (see Appendix B)-but it does not work for our main result. Though, in their framework, $R$ is not required to be noncollapsing, $R$ actually plays the role of a noncollapsing system with respect to $\lambda$. The key fact in their proof is that $R$ has base type but not higher type. Thus, if a mixed term $M$ has a TRS context as outermost layer, then any term reduced from $M$ has a base type. In particular, it will have no $\lambda$ symbol at the root after any reduction. Hence, $R$-reductions cannot create a new $\beta$-redex in a mixed term, just like noncollapsing systems. It is clear that $\lambda$ is left-linear and confluent. Thus, by taking $R$ and $\lambda$ as $R_{0}$ and $R_{1}$, respectively, in Conjecture $\mathrm{A}^{\prime}$, we can observe very similar proof diagrams when comparing their proof and our proof of Conjecture $\mathrm{A}^{\prime}$.

We now give a brief outline of the contents of the paper. Section 2 fixes some standard terminology and notation. Section 3 introduces an underlining device, necessary to keep track of subterms in a reduction. Section 4 considers "mixed terms," that is, terms obtained from the disjoint union of two TRSs. Several basic notions for mixed terms are introduced. The main lemma of this section (4.8.5) has a complicated proof, that is postponed to Appendix A. In Section 5 , we prove the main result of this paper. In order not to interrupt the flow of arguments, the long proof of one crucial proposition (5.2) is deferred to Section 6 .

1.1. Preliminaries. We assume that the reader is familiar with the basic concepts and notations concerning term rewriting systems (TRSs); otherwise, see the basic references mentioned in the Introduction. In this section, we exhibit the notions and concepts that are specific for the present paper, and we briefly recapitulate some of the more basic concepts.

A term rewriting system $R$ has an alphabet consisting of a (possibly infinite) set $\mathscr{F}$ of function symbols $F, G, H, \ldots$, each having an "arity," that is, the number of arguments that the function symbol requires, and a set of variables $x, y, z, \ldots$. So if $F$ is $n$-ary, then $F\left(t_{1}, \ldots, t_{n}\right)$ is a term, for terms $t_{1}, \ldots, t_{n}$. Constants are 0 -ary function symbols. The set of terms of $R$, notation $\operatorname{Ter}(R)$, contains the terms which are inductively generated from the constant symbols, the variables $x, y, z, \ldots$ and the other function symbols. Terms are denoted by $t, s, \ldots$ but occasionally also by $M, N, \ldots$. 
A TRS $R$ has a (possibly infinite) set $\operatorname{Red}(R)$ of reduction or rewrite rules $r$ : $t \rightarrow s$, or $t \rightarrow_{r} s$, where $r$ is the name of the rewrite rule. We adopt the usual restriction that the left-hand side $t$ is not a variable, and that the variables occurring in $s$, also occur in $t$. A rewrite step has the form $C\left[t^{\sigma}\right] \rightarrow_{r} C\left[s^{\sigma}\right]$, where $\sigma$ is a substitution and $C[$ ] a context, that is, a term with a "hole" $\square$. A substitution $\sigma$ is a homomorphism from $\operatorname{Ter}(R)$ to $\operatorname{Ter}(R)$, satisfying $F\left(t_{1}, \ldots, t_{n}\right)^{\sigma}=F\left(t_{1}^{\sigma}, \ldots, t_{n}^{\sigma}\right)$. The transitive reflexive closure of $\rightarrow_{r}$ is $\rightarrow_{r}$; the transitive closure of $\rightarrow_{r}$ is $\rightarrow_{r}^{+}$. The reflexive closure of $\rightarrow_{r}$ is $\rightarrow_{r}^{\underline{\equiv}}$. The convertibility (i.e., equivalence relation) generated by $\rightarrow_{r}$ is $=_{r}$. Often the subscript $r$ is omitted. Convertibility $(=)$ should not be confused with $\equiv$, which denotes syntactical equality. The notation $t \rightarrow^{n} s$ is short for $t \rightarrow \cdots \rightarrow s$ in $n$ steps.

We say that $t$ is a normal form if there is no $s$ such that $t \rightarrow s$. Further, $s$ has a normal form $t$ if $s \rightarrow t$ and $t$ is a normal form. The normal form of $t$ is denoted by $t \downarrow$ if it exists uniquely.

The concepts of confluence (also called the Church-Rosser property) and termination (also called the Strong Normalization property, stating that all reduction sequences must end eventually) are defined as usual. We say that $t$ is terminating if every reduction sequence starting with $t$ eventually must terminate. A TRS is "complete" if its reduction relation is confluent and terminating (this is also called canonical in the literature). A TRS $R$ is left-linear if $R$ contains no rewrite rule $t \rightarrow s$ such that $t$ contains two or more occurrences of the same variable.

We write $t \subseteq s$ to indicate that $t$ is a subterm of $s$. When referring to subterms, we will always mean specific occurrences of those subterms; we will however not need a more precise formalism to indicate occurrences (e.g., as sequence numbers). If $t \subseteq s$ and $t \not \equiv s$, we write $t \subset s$, and call $t$ a proper subterm of $s$.

\section{Underlined Reduction and Frozen Subterms}

Consider the TRS with set of reduction rules $\{F(x, C) \rightarrow x, F(C, x) \rightarrow x$, $H(x) \rightarrow x, G(x) \rightarrow x\}$ and the term $M \equiv F(H(C), G(C))$. Figure 1(a) displays the node-labeled tree corresponding to $M$. The term $M$ has the following reductions to its normal form:

(1) $M \rightarrow F(C, G(C)) \rightarrow G(C) \rightarrow C$

(2) $M \rightarrow F(H(C), C) \rightarrow H(C) \rightarrow C$.

Although both reductions end in $C$, the two $C$ 's are different with respect to their occurrence in $M$. This is graphically expressed in Figure 1(b) where the arrows indicate to which occurrence of $C$ the term $M$ is "collapsed." In the sequel, we will need to be precise about such reductions to occurrences of subterms, rather than mere subterms. Therefore, we introduce the concepts of "underlined" reductions and "frozen" subterms, as follows:

\section{Definition 2.1}

(i) Let $R$ be some TRS. Then $R_{e}$ is the TRS having as alphabet (or signature): the alphabet of $R$ extended with a new unary function symbol ' $e$.' The rewrite rules of $R_{e}$ are: those of $R$ extended with $e(x) \rightarrow x$. 


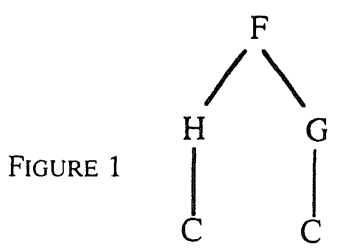

(a)



(b)

(ii) We write $\rightarrow_{e}$ for one $e$-reduction step, that is, a reduction step using the rule $e(x) \rightarrow x$. Thus: $C[e(M)] \rightarrow_{e} C[M]$ for a context $C[$ ] and a term $M$ in $R_{e}$.

(iii) For terms $M_{1}, M_{2}$ of $R_{e}$, we write $M_{1} \rightarrow_{\mathrm{f}} M_{2}$ ("f" for "frozen") if the redex contracted is not an $e$-redex nor in the scope of some ' $e$.' So if $C[e(P)] \rightarrow_{\mathrm{f}}^{S} N$ where $S$ is the contracted redex, then it is not the case that $S \subseteq e(P)$.

\section{Notation 2.2}

(i) For notational ease we will henceforth write $\underline{M}$ instead of $e(M)$ and $\underline{R}$ instead of $R_{e}$. Terms from $\underline{R}$ are "underlined" terms (even if they contain no actual underlining).

(ii) We write $\rightarrow$ for the transitive-reflexive closure of $\rightarrow_{f} \cup \rightarrow_{e}$. (This is, in fact, an ambiguous use of $\rightarrow$, since it was already in use for not underlined terms. But the present extension of the old $\rightarrow$ to the case of underlined terms will cause no confusion.)

(iii) In the sequel, $C\left[\underline{P}_{1}, \ldots, \underline{P}_{p}\right]$ denotes a term such that all underlinings are displayed, that is, $C\left[P_{1}, \ldots, P_{p}\right]$ contains no underlined subterm.

\section{Example 2.3}

(i) Let $R$ be the TRS as in the introduction of this section. Then, the $R$-term $F(H(C), G(\underline{C}))$ (in the e-notation: $F(e(H(C)), G(e(C)))$ has the reduction:

$$
\begin{aligned}
& F(\underline{H(C)}, G(\underline{C})) \rightarrow_{e} F(H(C), G(\underline{C})) \rightarrow_{\mathrm{f}} F(C, G(\underline{C})) \rightarrow_{\mathrm{f}} \\
& G(\underline{C}) \rightarrow_{\mathrm{f}} \underline{C} \rightarrow_{e} C .
\end{aligned}
$$

(ii) Note that the terms $F(H(C), G(C))$ and $F(H(C), G(C))$ are normal forms with respect to $\rightarrow_{\mathrm{f}}$ (f-normal forms).

Propositron 2.4. Let $R$ be a confluent and left-linear TRS. Then:

(i) the reduction $\rightarrow_{f}$ is confluent in $\underline{R}$ (See diagram in figure $2(a)$.)

(ii) the reductions $\rightarrow_{e}$ and $\rightarrow_{f}$ commute in $\underline{R}$. (See diagram in figure $2(b)$.)

Proof. Before starting the proof, note that the shaded arrows have the usual existential meaning. For example, the diagram in Figure 2(a) expresses:

$$
\forall M_{1}, M_{2}, M_{3} \exists M_{4}\left(M_{1} \rightarrow_{\mathrm{f}} M_{2} \& M_{1} \rightarrow_{\mathrm{f}} M_{3} \Rightarrow M_{2} \rightarrow_{\mathrm{f}} M_{4} \& M_{3} \rightarrow_{\mathrm{f}} M_{4}\right) .
$$

(i) Consider in $M_{1}$ the maximal underlined subterms. Here "maximal" refers to the subterm ordering $\subseteq$. Replace these subterms by mutually different new variables, in order to "code" these subterms. Do this everywhere in 


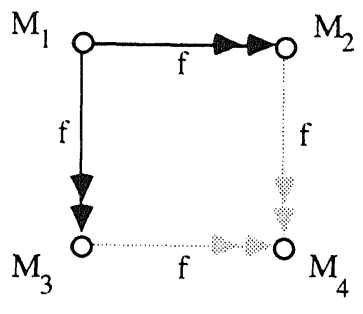

(a)

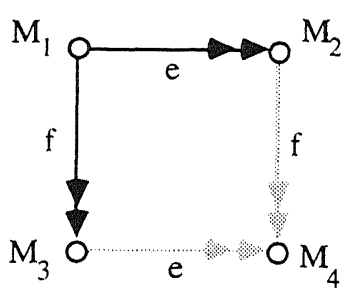

(b)

FIGURE 2

the reductions $M_{1} \rightarrow_{\mathrm{f}} M_{i}, i=2,3$. The resulting reductions $M_{1}^{*} \rightarrow M_{i}^{*}$, $i=2,3$, are "ordinary" (not underlined) reductions in $R$. Take the common reduct $M_{4}^{*}$ according to $R$; and replace in $M_{i}^{*} \rightarrow M_{4}^{*}(i=2,3)$ the coding variables by the original underlined subterms.

(ii) It suffices to prove the statement for the case that $M_{1} \rightarrow_{e} M_{2}$ is one step $M_{1} \rightarrow_{e} M_{2}$. Let this step be in fact $M_{1} \equiv C[\underline{N}] \rightarrow_{e} C[N] \equiv M_{2}$. Then $M_{3} \equiv C^{\prime}[\underline{N}, \ldots, \underline{N}]$ where all descendants of $\underline{N}$ are displayed. Now take $M_{4} \equiv C^{\prime}[\bar{N}, \ldots, \bar{N}]$. (Note that the reduction $M_{2} \rightarrow_{f} M_{4}$ is possible by left-linearity of $R$.)

We will be especially interested in reductions of the form $M \equiv C[\underline{P}] \rightarrow \underline{P}$ where $\underline{P}$ is the only underlined subterm in $C[\underline{P}]$. (Here, and in the sequel, we will permit ourselves a slight abuse of notation by using " $M \equiv C[\underline{P}] \rightarrow \underline{P}$ " for " $M \equiv C[P]$ and $C[\underline{P}] \rightarrow \underline{P}$. .") Graphically, the existence of such a reduction is indicated by an arrow as in Figure 3 (cf. the arrows in Figure 1(b). Indeed the two arrows there correspond with the $\rightarrow_{f}$-reductions:

$$
\begin{aligned}
& M \equiv F(H(C), G(\underline{C})) \rightarrow_{\mathrm{f}} F(C, G(\underline{C})) \rightarrow_{\mathrm{f}} G(\underline{C}) \rightarrow_{\mathrm{f}} \underline{C} \\
& \left.M \equiv F(H(\underline{C}), G(C)) \rightarrow_{\mathrm{f}} F(H(\underline{C}), C) \rightarrow_{\mathrm{f}} H(\underline{C}) \rightarrow_{\mathrm{f}} \underline{C}\right) .
\end{aligned}
$$

In the situation of Figure 3, we will sometimes say that (the displayed occurrence of) $P$ can be "pulled up from $M$." We will also say that $M$ "collapses to (the displayed occurrence of) $P$ ".

Remark 2.5. Since in $C[\underline{P}] \rightarrow \underline{P}$ the subterm $P$ initially is "frozen," it might be thought that $C[\underline{P}] \rightarrow \underline{P}$ implies $C[z] \rightarrow z$ for a fresh variable $z$. This is not the case as the following example shows: Let $R$ have the reduction rules

$$
\begin{aligned}
F(x) & \rightarrow G(x, x) \\
G(C, x) & \rightarrow x \\
H(x) & \rightarrow x .
\end{aligned}
$$

Then $F(H(C)) \rightarrow H(C)$ in view of the reduction sequence

$$
F(\underline{H(C)}) \rightarrow G(\underline{H(C)}, \underline{H(C)}) \rightarrow G(H(C), \underline{H(C)}) \rightarrow G(C, \underline{H(C)}) \rightarrow \underline{H(C)} .
$$

However, $F(z) \rightarrow z$ does not hold. The explanation is that in a reduction $C[P] \rightarrow \underline{P}$ not all descendants of the initial $\underline{P}$ need to remain frozen; only the $\underline{P}$ on the "main line" of descendants leading to the ultimate $\underline{P}$ in the right-hand side of $C[\underline{P}] \rightarrow \underline{P}$ must be frozen. As the above reduction sequence 
FIGURE 3

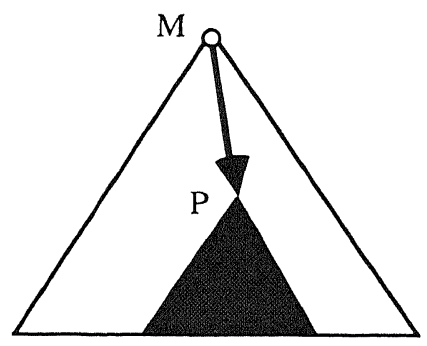

shows, some descendants of the initial $\underline{P}$ in $C[\underline{P}]$, not in the main line of descendants, may actually play a necessary role in the collapse to the ultimate $\underline{P}$. (What does hold is the implication $C[\underline{P}] \rightarrow_{\mathrm{f}} \underline{P} \Rightarrow C[z] \rightarrow z$ for a fresh variable $z$. The next proposition (part (i)) generalizes this obvious fact.)

Proposition 2.6. Let $R$ be a confluent and left-linear TRS. Then:

(i) $C[\underline{P}] \rightarrow_{f} C^{\prime}[\underline{P}, \ldots, \underline{P}] \Leftrightarrow$

$C[\bar{z}] \rightarrow C^{\prime}[z, \ldots, z]$ for a fresh variable $z \Leftrightarrow$

$C[Q] \rightarrow_{f} C^{\prime}[Q, \ldots, Q]$ for all $Q$.

(ii) Let $C[\underline{P}] \rightarrow_{f} C^{\prime}[\underline{\underline{P}}, \ldots, \underline{P}]$ and $P \equiv C^{\prime \prime}[Q]$. Then $C\left[C^{\prime \prime}[\underline{Q}]\right] \rightarrow_{f}$ $C^{\prime}\left[C^{\prime \prime}[\underline{Q}], \ldots, C^{\prime \prime}[\underline{Q}]\right]$.

ProOF. Routine.

Proposition 2.7. Let $R$ be a confluent and left-linear TRS. Let $C[\underline{P}, \ldots, \underline{P}]$ $\rightarrow^{k} \underline{P}$ (i.e., a reduction of $k$ steps of $\rightarrow_{e}$ or $\rightarrow_{f}$ ). Then for some occurrence of $\underline{P}$ in $C[\underline{P}, \ldots, \underline{P}]$ and some $k^{\prime} \leq k$ :

$$
C[P, \ldots, \underline{P}, \ldots, P] \rightarrow^{k^{\prime}} \underline{P} .
$$

Proof. Consider a reduction $C[\underline{P}, \ldots, \underline{P}] \rightarrow^{k} \underline{P}$. Now the final $\underline{P}$ can be traced back to a unique ancestor $\underline{P}$ in $C[\underline{P}, \ldots, \underline{P}]$. Removing the underlining of the other $\underline{P}$ in $C[\underline{P}, \ldots, \underline{P}]$ we obtain $C[P, \ldots, \underline{P}, \ldots, P]$. Clearly, there is now a reduction $C[P, \ldots, \underline{P}, \ldots, P] \rightarrow \underline{P}$ which is the "same" as the original reduction $C[\underline{P}, \ldots, \underline{P}, \ldots, \underline{P}] \rightarrow \underline{P}$ except that we possibly gain some $e$-steps (removals of underlinings).

LEMMA 2.8. Let $R$ be a confluent and left-linear TRS. Let $C[\underline{P}] \rightarrow \underline{P}$ and $Q \rightarrow P$. Then $C[\underline{Q}] \rightarrow \underline{Q}$.

Proof. Suppose $C[\underline{P}] \rightarrow^{k} \underline{P}$. We will prove the lemma by induction on $k$. The case $k=0$ is trivial. Now let

$$
C[\underline{P}] \rightarrow C^{\prime}[\underline{P}, \ldots, \underline{P}] \rightarrow^{k-1} \underline{P} .
$$

By Proposition 2.7 , we have for some occurrence of $\underline{P}$ in $C^{\prime}[\underline{P}, \ldots, \underline{P}]$ and some $k^{\prime} \leq k-1$ :

$$
C^{\prime}[P, \ldots, \underline{P}, \ldots, P] \rightarrow^{k^{\prime}} \underline{P} .
$$

By the induction hypothesis, $C^{\prime}[P, \ldots, \underline{Q}, \ldots, P] \rightarrow \underline{Q}$. So we have

$$
\begin{aligned}
C[\underline{Q}] & \rightarrow C^{\prime}[\underline{Q}, \ldots, \underline{Q}, \ldots, \underline{Q}] \rightarrow C^{\prime}[Q, \ldots, \underline{Q}, \ldots, Q] \\
& \rightarrow C^{\prime}[P, \ldots, \underline{Q}, \ldots, P] \rightarrow \underline{Q} .
\end{aligned}
$$



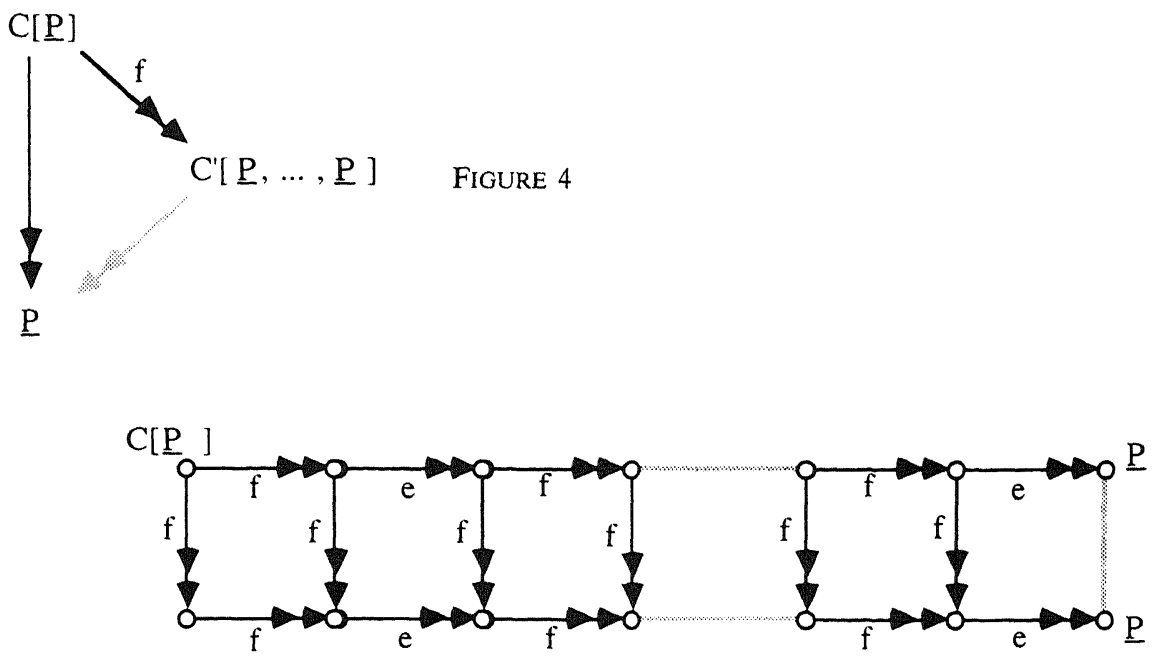

$C^{\prime}[\underline{P}, \ldots, \underline{P}]$

Figure 5

Proposition 2.9. Let $R$ be a confluent and left-linear TRS. Let $C[\underline{P}] \rightarrow \underline{P}$ and let $C[\underline{P}] \rightarrow{ }_{f} C^{\prime}[\underline{P}, \ldots, \underline{P}]$, where all occurrences of $\underline{P}$ in $C^{\prime}[\underline{P}, \ldots, \underline{P}]$ are displayed.

Then $C^{\prime}[\underline{P}, \ldots, \underline{P}]$ contains at least one occurrence of $\underline{P}$ and $C^{\prime}[\underline{P}, \ldots, \underline{P}] \rightarrow \underline{P}$ (see Figure 4).

Proof. That $C^{\prime}[\underline{P}, \ldots, \underline{P}]$ contains some occurrence of $\underline{P}$ follows immediately from $C^{\prime}[\underline{P}, \ldots, \underline{P}] \rightarrow \underline{P}$, since underlinings cannot be created during a reduction.

The proof of $C^{\prime}[\underline{P}, \ldots, \underline{P}] \rightarrow \underline{P}$ follows from the diagram in Figure 5. Note that the given reduction $C[\underline{P}] \rightarrow \underline{P}$ consists of some sequence of $\rightarrow_{\mathrm{f}}$ and $\rightarrow_{\mathrm{e}}$ reductions; it is displayed in the upper part of the diagram in Figure 5.

This diagram construction is possible by Proposition 2.4. Note that the right-hand side of the diagram is the empty reduction $\underline{P} \rightarrow_{\mathrm{f}} \underline{P}$ (i.e., consisting of zero steps), since $\underline{P}$ is an f-normal form. Hence, the lower side of the reduction diagram gives us a reduction $C^{\prime}[\underline{P}, \ldots, \underline{P}] \rightarrow \underline{P}$.

LEMMA 2.10. Let $R$ be a confluent and left-linear TRS.

If $C[\underline{P}] \rightarrow \underline{P}$ and $P \rightarrow Q$, then $C[\underline{Q}] \rightarrow \underline{Q}$.

ProOF. Suppose $C[\underline{P}] \rightarrow^{k} \underline{P}$. We will prove the proposition by induction on $k$. The case $k=0$ is trivial: then $C[\underline{P}] \equiv \underline{P}$ and indeed $Q \rightarrow Q$. Induction hypothesis: the statement holds for $k-1(k>0)$. Now let $C[\underline{P}] \rightarrow{ }^{k} \underline{P}$. So $C[\underline{P}] \rightarrow C^{\prime}[\underline{P}, \ldots, \underline{P}] \rightarrow^{k-1} \underline{P}$. By Proposition 2.7 , we have a reduction $C^{\prime}[P, \ldots, \underline{P}, \ldots, P] \rightarrow{ }^{k^{\prime}} \underline{P}$ for some $k^{\prime} \leq k-1$ and for some occurrence of $P$. Hence, by the induction hypothesis, $C^{\prime}[P, \ldots, Q, \ldots, P] \rightarrow Q$.

By Proposition 2.9 , since $C^{\prime}[P, \ldots, Q, \ldots, P] \rightarrow_{\mathrm{f}} C^{\prime}[Q, \ldots, Q, \ldots, Q]$, we have $C^{\prime}[Q, \ldots, Q, \ldots, Q] \rightarrow Q$. Concatenating this reduction with $C[Q] \rightarrow$ $C^{\prime}[\underline{Q}, \ldots, \underline{Q}, \ldots, \underline{Q}] \rightarrow C^{\prime}[Q, \ldots, \underline{Q}, \ldots, Q]$, we have indeed $C[\underline{Q}] \rightarrow \underline{Q}$. 
Remark 2.11. From the preceding propositions we see that the relation $C[\underline{P}] \rightarrow \underline{P}$ is preserved under convertibility $(=$, the equivalence generated by $\rightarrow$, that is, by $\rightarrow_{\mathrm{e}}, \rightarrow_{\mathrm{f}}$.). For, combining Lemma's 2.8 and 2.10, we have:

$$
C[\underline{P}] \rightarrow \underline{P} \quad \& \quad P=Q \Rightarrow C[\underline{Q}] \rightarrow \underline{Q} .
$$

Moreover, $C[\underline{P}] \rightarrow \underline{P}$ is preserved under any reduction of $C[\underline{P}]$ which leaves $\underline{P}$ unaffected, as Proposition 2.9 states ( $\underline{P}$ may be multiplied, though.)

\section{Mixed Terms}

We will now consider disjoint unions, or as we will call them, direct sums $R_{\mathrm{b}} \oplus R_{\mathrm{w}}$ of TRSs $R_{\mathrm{b}}, R_{\mathrm{w}}$ with disjoint alphabets. Henceforth, we assume that $R_{\mathrm{b}}, R_{\mathrm{w}}$ are left-linear and complete. Let $\mathscr{F}$ be a set of function and constant symbols, and let $\mathscr{V}$ be a countably infinite set of variables. Then $\operatorname{Ter}(\mathscr{F}, \mathscr{V})$ is the set of terms constructed from $\mathscr{F}$ and $\mathscr{V}$. If $R_{i}(i=\mathrm{b}, \mathrm{w})$ are TRSs with rule sets $\operatorname{Red}\left(R_{i}\right)$, terms $\operatorname{Ter}\left(\mathscr{F}_{\mathrm{i}}, \mathscr{V}\right)$ such that $\mathscr{F}_{\mathrm{b}}$ and $\mathscr{F}_{\mathrm{w}}$ are disjoint, then $R_{\mathrm{b}} \oplus R_{\mathrm{w}}$ is the TRS with terms $\operatorname{Ter}\left(\mathscr{F}_{\mathrm{b}} \cup \mathscr{F}_{\mathrm{w}}, \mathscr{V}\right)$ and reduction rules $\operatorname{Red}\left(R_{\mathrm{b}}\right) \cup$ $\operatorname{Red}\left(R_{\mathrm{w}}\right)$. Instead of $\operatorname{Ter}\left(\mathscr{F}_{\mathrm{b}} \cup \mathscr{F}_{\mathrm{w}}, \mathscr{V}\right)$ we will also write $\operatorname{Ter}\left(R_{\mathrm{b}} \oplus R_{\mathrm{w}}\right)$.

As a mnemonic device, we will call the function and constant symbols of $R_{\mathrm{b}}$ black and those of $R_{\mathrm{w}}$ white. To distinguish in print between them, the black symbols are capitals and the white symbols are lower case. Thus, a term $M \in \operatorname{Ter}\left(R_{\mathrm{b}} \oplus R_{\mathrm{w}}\right)$, in its tree notation, is a constellation of black and white "triangles," as in Figure 6. Here, the root of $M$ is the leading symbol of $M$.

Note that if $R_{\mathrm{b}}$ and $R_{\mathrm{w}}$ are complete (as always assumed in this paper), then every term in $\operatorname{Ter}\left(R_{\mathrm{b}} \oplus R_{\mathrm{w}}\right)$ has a normal form. This can be easily proved using innermost reductions (in which the only redexes reduced are those containing no proper subredexes). Moreover, the normal form is unique, since $R_{\mathrm{b}} \oplus R_{\mathrm{w}}$ is confluent (by the main theorem in Toyama [1987a]). The normal form of term $t$ will be denoted by $t \downarrow$.

\section{Definition 3.1}

(i) Let $M \equiv C\left[B_{1}, \ldots, B_{n}\right] \in \operatorname{Ter}\left(R_{\mathrm{b}} \oplus R_{\mathrm{w}}\right)$ and $C[] \not \equiv \square$. Then we write $M \equiv C \llbracket B_{1}, \ldots, B_{n} \rrbracket$ if $C[, \ldots$,$] is a context of R_{\mathrm{b}}$ and $\operatorname{root}\left(B_{i}\right) \in \mathscr{F}_{\mathrm{w}}$ for $i=1, \ldots, n$. (Likewise with $\mathrm{b}$, w interchanged.) The $B_{i}$ are called the principal subterms of $M$.

(ii) The set $\mathbf{S}(M)$ of special subterms (more precisely, subterm occurrences) is inductively defined as follows:

$$
\mathbf{S}(M)=\left\{\begin{array}{lll}
\{M\} & \text { if } \quad M \in \operatorname{Ter}\left(R_{d}\right)(d=\mathrm{b}, \mathrm{w}) \\
\{M\} \cup \bigcup_{i} \mathbf{S}\left(B_{i}\right) & \text { if } \quad M \equiv C \llbracket B_{1}, \ldots, B_{n} \rrbracket(n>0),
\end{array}\right.
$$

(iii) $\mathbf{S}_{d}(M)=\left\{N \mid N \in \mathbf{S}(M)\right.$ and $\left.\operatorname{root}(N) \in \mathscr{F}_{d}\right\}(d=\mathrm{b}$,w).

(iv) $\mathbf{G}_{d}(M)=\left\{N \mid M \rightarrow N\right.$ and $\left.\operatorname{root}(N) \in \mathscr{F}_{d}\right\}(d=\mathrm{b}, \mathrm{w})$.

Definition 3.2. Let $M \in \operatorname{Ter}\left(R_{\mathrm{b}} \oplus R_{\mathrm{w}}\right)$. Then:

$$
\operatorname{rank}(M)= \begin{cases}1 & \text { if } \quad M \in \operatorname{Ter}\left(R_{d}\right)(d=\mathrm{b}, \mathrm{w}) \\ \max _{i}\left\{\operatorname{rank}\left(B_{i}\right)\right\}+1 & \text { if } \quad M \equiv C \llbracket B_{1}, \ldots, B_{n} \rrbracket(n>0)\end{cases}
$$




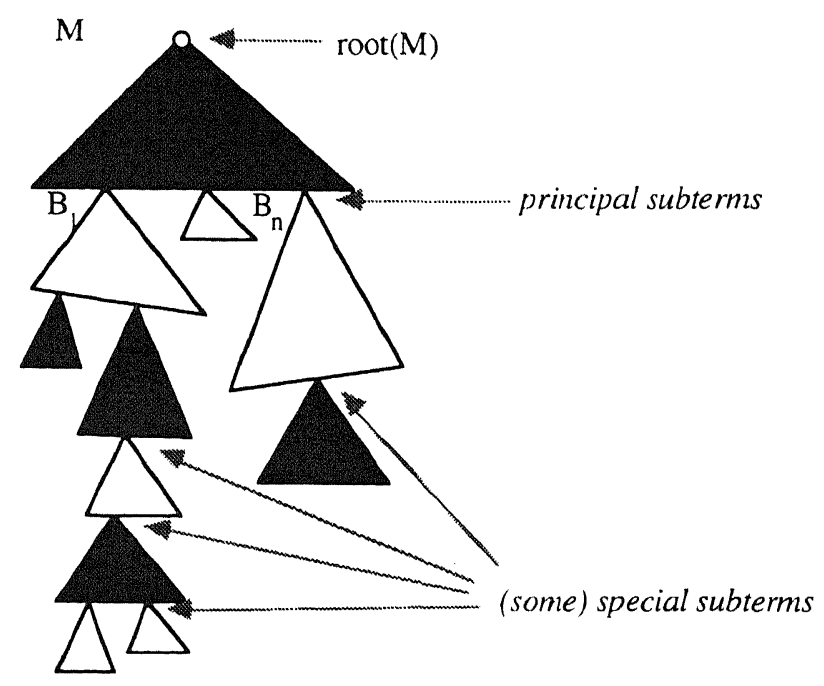

FIGURE 6

The following fact (where $\rightarrow$ is reduction in $R_{\mathrm{b}} \oplus R_{\mathrm{w}}$ ) has a routine proof that is omitted.

Proposition 3.3. If $M \rightarrow N$, then $\operatorname{rank}(M) \geq \operatorname{rank}(N)$.

Proposition 3.4. Let $M \rightarrow N$ where both $M, N$ have a black root. Then there exists a reduction $M \equiv M_{0} \rightarrow M_{1} \rightarrow M_{2} \rightarrow \cdots \rightarrow M_{n} \equiv N$ such that all $M_{i}(i=$ $0, \ldots, n)$ have a black root.

Proof. Let $M \rightarrow{ }^{k} N(k \geq 0)$. We will prove the proposition by induction on $k$. The case $k=0$ is trivial. Now let $M \rightarrow M^{\prime} \rightarrow^{k-1} N$. If the root of $M^{\prime}$ is black, then we are through, by the induction hypothesis. If the root of $M^{\prime}$ is white, then there exists a context $C\left[\right.$ ] with black root such that $M \equiv C\left[M^{\prime}\right]$ and $C[] \rightarrow \square$, the trivial context. Thus, we have a reduction $M \equiv C\left[M^{\prime}\right] \rightarrow$ $C[N] \rightarrow N$ in which all terms have a black root.

LEMMA 3.5. Let $M \rightarrow N$. Let $Q$ be a special subterm of $N$ with white (black) root. Then there is a special subterm $P$ of $M$ with white (black) root such that $P \equiv Q$ or $P \rightarrow Q$.

Proof. Since $M \rightarrow N$, there exists a rewriting rule $C_{1}\left[x_{1}, \ldots, x_{n}\right] \rightarrow$ $C_{r}\left[x_{i 1}, \ldots, x_{i m}\right]$, a context $C[]$, and mixed terms $M_{1}, \ldots, M_{n}$ such that $M \equiv$ $C\left[C_{1}\left[M_{1}, \ldots, M_{n}\right]\right]$ and $N \equiv C\left[C_{r}\left[M_{i 1}, \ldots, M_{i m}\right]\right]$ where $M_{i 1}, \ldots, M_{i m} \in$ $\left\{M_{1}, \ldots, M_{n}\right\}$.

Case 1. $Q$ and $C_{r}\left[M_{i 1}, \ldots, M_{i m}\right]$ are disjoint. $Q$ occurs in the context $C[]$ as a special subterm. Thus, we can take $Q$ as $P$ in $M \equiv C\left[C_{1}\left[M_{1}, \ldots, M_{n}\right]\right]$.

Case 2. $Q \subset C_{r}\left[M_{i 1}, \ldots, M_{i m}\right]$. As $Q$ is a special and proper subterm occurrence of $C_{r}\left[M_{i 1}, \ldots, M_{i m}\right], Q$ must occur in some $M_{i j}$. Since $M_{i j} \in$ $\left\{M_{1}, \ldots, M_{n}\right\}$, we can take $Q$ as $P$ in $M$ as a special subterm.

Case 3. $Q \equiv C_{\mathrm{r}}\left[M_{i 1}, \ldots, M_{i m}\right]$. If $C_{\mathrm{l}}\left[M_{1}, \ldots, M_{n}\right]$ has a white root, then take $C_{\mathrm{l}}\left[M_{1}, \ldots, M_{n}\right]$ in $M$ as $P$. It is clear that $P \rightarrow Q$ and $P$ is a special 

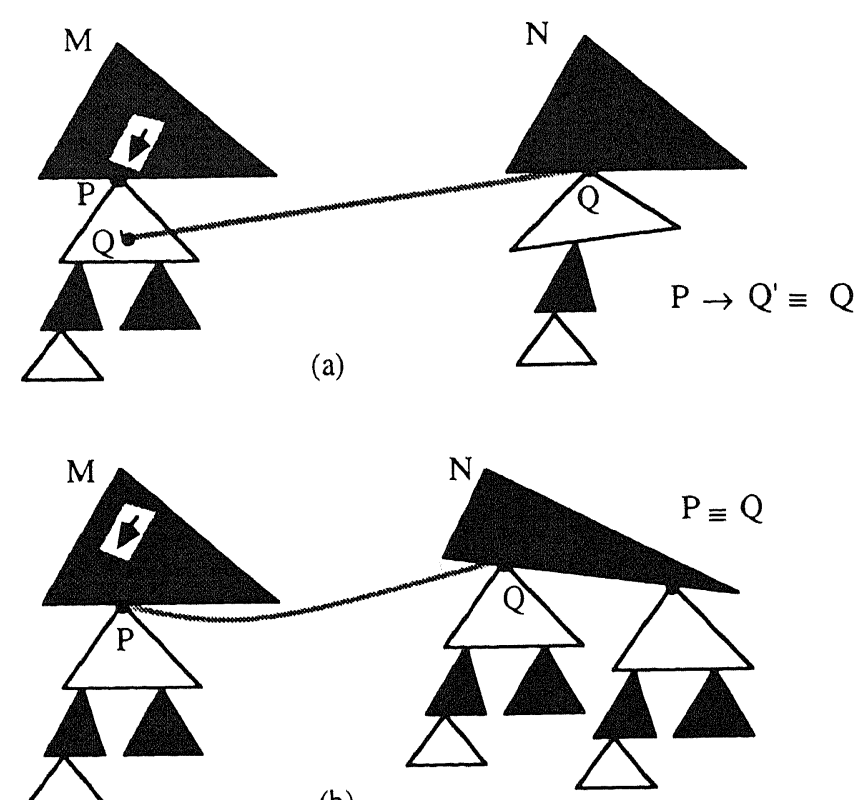

(b)

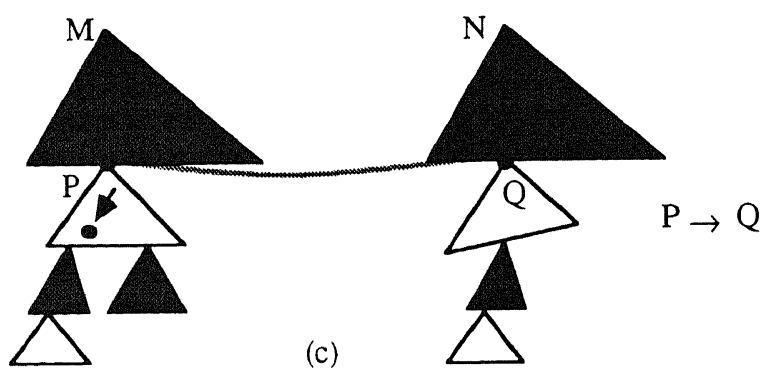

Figure 7

subterm with white root (see Figure $7(\mathrm{a})$ ). If $C_{\mathrm{l}}\left[M_{1}, \ldots, M_{n}\right]$ has a black root then $C_{\mathrm{r}}[, \ldots,] \equiv \square$ and $Q$ must be in $\left\{M_{1}, \ldots, M_{n}\right\}$, say $Q \equiv M_{p}$. Thus, we can take $M_{p}$ in $M$ as a special subterm (see Figure 7(b)).

Case 4. $C_{\mathrm{r}}\left[M_{i 1}, \ldots, M_{i m}\right] \subset Q$. Let $Q \equiv C_{a}\left[C_{\mathrm{r}}\left[M_{i 1}, \ldots, M_{i m}\right]\right]$ where $C_{a}[, \ldots,] \not \equiv \square$. Then there exists a context $C_{b}\left[\right.$ ] such that $N \equiv C_{b}[Q] \equiv$ $C_{b}\left[C_{a}\left[C_{\mathrm{r}}\left[M_{i 1}, \ldots, M_{i m}\right]\right]\right]$ and $M \equiv C_{b}\left[C_{a}\left[C_{1}\left[M_{1}, \ldots, M_{n}\right]\right]\right]$. Take $P \equiv$ $C_{a}\left[C_{1}\left[M_{1}, \ldots, M_{n}\right]\right]$ in $M$. Then, $P \rightarrow Q$ and $P$ is a special subterm with white root of $M$ (see Figure $7(\mathrm{c})$ ).

LEMMA 3.6. Let $M$ have a black root $\left(\in \mathbf{F}_{b}\right)$ and suppose $M \rightarrow N$ where $N$ has a white root. Then $M$ has a special subterm $P$ with white root such that $M \equiv C[\underline{P}] \rightarrow \underline{P}$ and $P \rightarrow N$. (See Figure 8.)

Proof. Suppose $M \rightarrow^{k} N$. We will prove the proposition by induction on $k$. The case $k=1$ is trivial; then $N$ must be in fact one of the principal subterms $M_{\mathrm{r}}$ of $M \equiv C^{\prime} \llbracket\left[M_{1}, \ldots, M_{\mathrm{r}}, \ldots, M_{n} \rrbracket\right.$ and we can take $P \equiv M_{\mathrm{r}}$.

Induction Hypothesis: Suppose the statement is proved for $k-1$. Now consider $M \rightarrow^{k} N$, that is, $M \rightarrow M^{\prime} \rightarrow^{k-1} N$ for some $M^{\prime}$. 


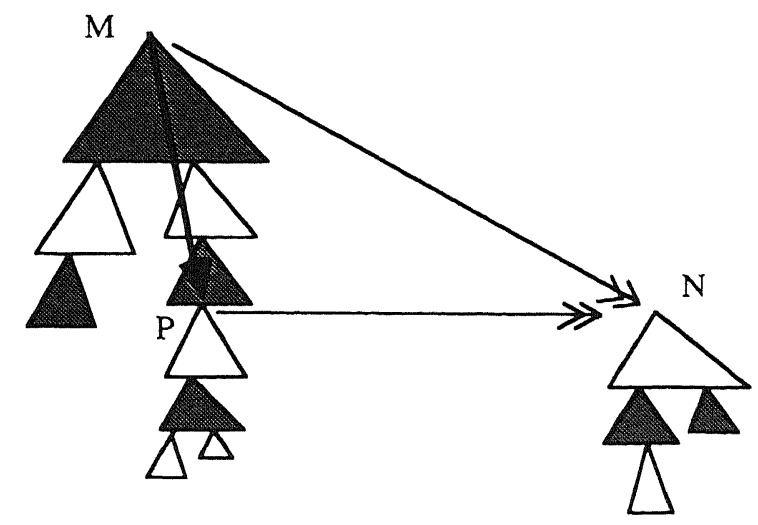

FIGURE 8

Case 1. The root of $M^{\prime}$ is white. Then $M \equiv C^{\prime} \llbracket\left[M_{1}, \ldots, M_{\mathrm{r}}, \ldots, M_{n} \rrbracket \rightarrow M^{\prime}\right.$ $\equiv M_{\mathrm{r}}$ for some r. Take $P \equiv M_{\mathrm{r}}$.

Case 2. The root of $M^{\prime}$ is black. According to the induction hypothesis $M^{\prime}$ has a special subterm $P^{\prime}$ with white root such that $M^{\prime} \equiv C\left[\underline{P}^{\prime}\right] \rightarrow \underline{P}^{\prime}$ and $P^{\prime} \rightarrow N$. By Lemma 3.5, there is a special subterm $P \in \mathrm{S}_{\mathrm{w}}(\bar{M})$ such that $P \rightarrow P^{\prime}$ or $P \equiv P^{\prime}$. We distinguish two subcases:

Case 2.1. $P \rightarrow P^{\prime}$. Then $M \equiv C[P] \rightarrow M^{\prime} \equiv C\left[P^{\prime}\right]$. By Lemma $2.8, M \equiv$ $C[\underline{P}] \rightarrow \underline{P}$. Since $P \rightarrow P^{\prime} \rightarrow N$ the statement is proved for this case.

Case 2.2. $P \equiv P^{\prime}$. Then $M \equiv C^{\prime}[\underline{P}] \rightarrow C^{*}[\underline{P}, \ldots, \underline{P}, \ldots, \underline{P}] \rightarrow_{e}$ $C^{*}[P, \ldots, \underline{P}, \ldots, P] \equiv M^{\prime} \equiv C[\underline{P}] \equiv C\left[\underline{P}^{\prime}\right] \rightarrow \underline{P}^{\prime} \equiv \underline{P}$.

3.7. Essential Subterms. As the last lemma (3.6) states, if $M$ has a black root all reductions of $M$ to a term with white root can be "factored through" reductions of $M$ to its special subterms with white root. Of these special subterms with white root, some are even more special: the essential subterms of $M$. As we will see, every collapse reduction of $M$ to a special subterm $Q$ with white root can be factored as a collapse of $M$ to an essential subterm $P$ followed by a collapse of $P$ to $Q$. (See Figure 9.)

Definition 3.7.1. Let $M$ have a black root. Let $P$ be a special subterm of $M$ with white root such that $M$ collapses to $P$. Then $P$ is an essential subterm (occurrence) of $M$ if there is no special subterm $P^{\prime}$ with white root such that $P \not \equiv P^{\prime}, M$ collapses to $P^{\prime}$, and $P^{\prime}$ collapses to $P$. The set of essential subterms of $M$ is $\mathbf{E}(M)$. (Likewise, with colors interchanged.)

In other words: Let $\operatorname{root}(M) \in \mathscr{F}_{\mathrm{b}}$. Then the essential subterms of $M$ are the maximal elements in the set $\left\{N \in \mathbf{S}_{w}(M) \mid M\right.$ collapses to $\left.N\right\}$, partially ordered by the relation "... collapses to...".

LEMMA 3.7.2. Let $M$ have a black root, and suppose $M \rightarrow N$ where $N$ has a white root. Then for some essential subterm $P$ of $M: P \rightarrow N$.

Proof. Immediately by Lemma 3.6 and Definition 3.7.1. 
FIGURE 9



3.8. Deterministic Terms. In the preceding subsection, we have already set up some notions to discuss the "collapsing behavior" of mixed terms. We will now introduce an important property of this collapsing behavior-first for the case of a single TRS.

Definition 3.8.1. Let $R$ be a TRS and $M \in \operatorname{Ter}(R)$. Then $M$ is a nondeterministic term if

(i) $M \equiv C[P, Q]$ and $C[\underline{P}, Q] \rightarrow \underline{P}, C[P, Q] \rightarrow Q$ (Figure 10(a)), or

(ii) $M \equiv C[P], P \equiv C^{\prime}[Q], C[\underline{P}] \rightarrow \underline{P}, C\left[\bar{C}^{\prime}[\underline{Q}]\right] \rightarrow \underline{Q}$ but not $C^{\prime}[\underline{Q}] \rightarrow \underline{Q}$

(Figure 10(b)); here $P \rightarrow Q$ denotes that $P$ cannot collapse to $Q$ ).

An example of a nondeterministic term was given in the introduction of Section 2, for nondeterminism of type (i). As an example of nondeterminism of type (ii) consider $R=\{F(x) \rightarrow G(x, x), G(D, x) \rightarrow x, G(H(y), D) \rightarrow y, H(D)$ $\rightarrow D, C \rightarrow D$. This TRS is left-linear and complete. Now take $M \equiv F(H(C)$;



Remark 3.8.2. The phenomenon of nondeterministic terms is caused by ambiguities between the rewrite rules (i.e., the presence of "critical pairs"). Indeed, one can prove: In an orthogonal TRS (i.e., left-linear, non-ambiguous TRS) all terms are deterministic. The proof is rather lengthy and, since we have no need for this fact here, is not included in this paper.

Definition 3.8.3. Let $R_{\mathrm{b}}, R_{\mathrm{w}}$ be arbitrary TRSs and let $M \in \operatorname{Ter}\left(R_{\mathrm{b}} \oplus R_{\mathrm{w}}\right)$. Then $M$ is a mixed nondeterministic term if $M$ has at least two essential subterm occurrences. (See Figure 11.)

Remark 3.8.4. There are $R_{\mathrm{b}}, R_{\mathrm{w}}$ and terms $M, M^{\prime}$ with $M \rightarrow M^{\prime}$ such that $M^{\prime}$ is mixed nondeterministic, but $M$ is not. Example: consider $R_{\mathrm{b}}=\{G(x) \rightarrow$ $F(x, x), F(x, C) \rightarrow x, F(C, x) \rightarrow x\}, \quad R_{\mathrm{w}}=\{g(x) \rightarrow x\}$ and $M \equiv G(g(C)) \rightarrow$ $F(g(C), g(C)) \equiv M^{\prime}$.

Clearly, a mixed nondeterministic term is nondeterministic in the sense of Definition 3.8.1. In the sequel, we will say that a term $M$ has color change if $\operatorname{root}(M)$ is black and $\operatorname{root}(M \downarrow)$ is white, or vice versa. The following lemma plays an important role in Section 5. 


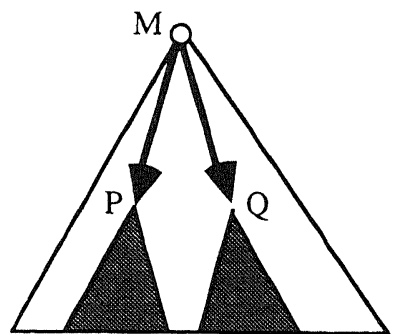

(a)

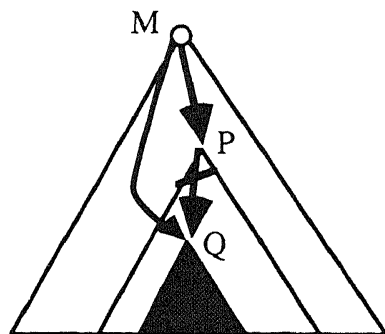

FIGURE 10

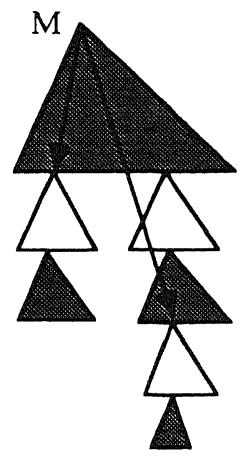

(a)



(b)

FIGURE 11

LEMMA 3.8.5. (MAIN LEMMA). Let $R_{b}, R_{w}$ be left-linear and complete. Let $M$ be terminating and let $M$ have color change. Then $M$ has exactly one essential subterm.

Proof. See Appendix A.

\section{Termination for the Direct Sum}

In this section, we will prove the main result, that is, the termination property for the direct sum $R_{\mathrm{b}} \oplus R_{\mathrm{w}}$ of left-linear and complete $R_{\mathrm{b}}, R_{\mathrm{w}}$. To this end, we define for a term $M \in \operatorname{Ter}\left(R_{\mathrm{b}} \oplus R_{\mathrm{w}}\right)$ two terms: the black projection $M^{\mathrm{b}} \in \operatorname{Ter}\left(R_{\mathrm{b}}\right)$ of $M$, and the white projection $M^{\mathrm{w}} \in \operatorname{Ter}\left(R_{\mathrm{w}}\right)$ of $M$. Roughly, the black/white projections of $M$ contain precisely the "information" in the black, respectively white, part of $M$. In fact, we will prove that if $M$ is a supposed minimal (with respect to length) term with white root, admitting an infinite reduction, then the white projection $M^{\mathrm{w}}$ has already an infinite reduction. As $M^{\mathrm{w}}$ is in $\operatorname{Ter}\left(R_{\mathrm{w}}\right)$, this is in contradiction with the termination property of $R_{\mathrm{w}}$ and we will have proved termination for $R_{\mathrm{b}} \oplus R_{\mathrm{w}}$.

VARIABLE CONVENTION 4.1. From now on we will assume that every term $M \in \operatorname{Ter}\left(R_{b} \oplus R_{w}\right)$ has only " $x$ " as variable occurrences, unless other variables are explicitly displayed. Since $R_{\mathrm{b}} \oplus R_{\mathrm{w}}$ is left-linear, this variable convention may be assumed in the sequel without loss of generality. 
While the definition and properties of the white projection $M^{\mathrm{w}}$ (the black projection $M^{\mathrm{b}}$ ) of $M$ will be given and proved in Section 5, it is sufficient for the rest of this section to assume the properties as stated in the following proposition.

PROPOSITION 4.2. Let every maximal special subterm with black (white) root of $M \in \operatorname{Ter}\left(R_{b} \oplus R_{w}\right)$ be terminating (if it exists). Then the white projection $M^{w}$ (the black projection $M^{b}$ ) of $M$ has the properties (where the black projection $M^{b}$ has the properties obtained by interchanging $w, b$ and white and black.):

(i) If $M \equiv x$, then $M^{w} \equiv x$.

(ii) If $M \equiv f\left(M_{1}, \ldots, M_{n}\right)(n \geq 0)$ has a white root, then $M^{w} \equiv f\left(M_{1}^{w}, \ldots, M_{n}^{w}\right)$.

(iii) If $M$ has a black root and an essential subterm $N$, then $M^{w} \rightarrow N^{w}$.

(iv) If $M \rightarrow x$, then $M^{w} \rightarrow x$.

Proof. See the next section.

Remark 4.3. Let $M \equiv C\left[M_{1}, \ldots, M_{n}\right]$ where $C[, \ldots$,$] is a context in$ $\operatorname{Ter}\left(R_{\mathrm{w}}\right)$. Then, from Proposition 4.2(i) and (ii), we have $M^{\mathrm{w}} \equiv C\left[M_{1}^{\mathrm{w}}, \ldots, M_{n}^{\mathrm{w}}\right]$. From this it follows that $M^{\mathrm{w}} \equiv M$ if $M \in \operatorname{Ter}\left(R_{\mathrm{w}}\right)$.

Remark 4.4. It is clear that the assumption "Every maximal special subterm with black root of $M \in \operatorname{Ter}\left(R_{b} \oplus R_{w}\right)$ is terminating (if it exists)" means that (i) if $M$ has a white root, every principal subterm (if it exists) is terminating and (ii) if $M$ has a black root, $M$ itself is terminating.

Remark 4.5. Let every maximal special subterm with black root of $M \in$ $\operatorname{Ter}\left(R_{\mathrm{b}} \oplus R_{\mathrm{w}}\right)$ be terminating, and let $M \rightarrow N$. Then, it is trivial that every maximal special subterm with black root of $N$ is also terminating. Thus, if $M$ has the white (black) projection by Proposition 4.2, then so has $N$.

Notation 4.6

(i) We write $M \equiv{ }_{0} N$ when $M, N$ have the same outermost-layer context, that is, $M \equiv C \llbracket M_{1}, \ldots, M_{m} \rrbracket$ and $N \equiv C \llbracket N_{1}, \ldots, N_{m} \rrbracket$ for some $M_{i}, N_{i}$ $(i=1, \ldots, m)$.

(ii) Let $M \equiv C \llbracket M_{1}, \ldots, M_{m} \rrbracket$ and suppose $M \rightarrow^{R} N$ (i.e., $N$ is obtained from $M$ by contracting the redex occurrence $R$ ). If the redex occurrence $R$ occurs in some $M_{i}$, we write $M \rightarrow_{\mathrm{i}} N$ ("inner reduction"); otherwise, we write $M \rightarrow{ }_{0} N$ ("outer reduction").

Note that $M_{1} \rightarrow_{\mathrm{i}} M_{2}, M_{2} \rightarrow_{\mathrm{i}} M_{3}$ implies $M_{1} \rightarrow_{\mathrm{i}} M_{3}$.

Proposition 4.7. Let $M \rightarrow{ }_{0} N$ where $M, N$ have white roots. Suppose $M \equiv_{0} A$ and $A \rightarrow_{i} M$ (internal reduction), where every maximal special subterm with black root of $A$ is terminating. Then there exists a term $B$ such that $N \equiv{ }_{0} B$, $A \rightarrow_{0} B, B \rightarrow_{i} N$ and $A^{w} \rightarrow B^{w}$. (See diagram in Figure 12.)

Proof. Let $A \equiv C \llbracket A_{1}, \ldots, A_{m} \rrbracket, \quad M \equiv C \llbracket M_{1}, \ldots, M_{m} \rrbracket$ and $N \equiv$ $C^{\prime} \llbracket\left[M_{i 1}, \ldots, M_{i n} \rrbracket\left(i_{j} \in\{1, \ldots, m\}\right)\right.$. Take $B \equiv C^{\prime} \llbracket\left[A_{i 1}, \ldots, A_{i n} \rrbracket\right.$. Then $A \rightarrow_{\mathrm{o}} B$ and $B \rightarrow_{\mathrm{i}} N$. From $A^{\mathrm{w}} \equiv C\left[A_{1}^{\mathrm{w}}, \ldots, A_{m}^{\mathrm{w}}\right]$ and $B^{\mathrm{w}} \equiv C^{\prime}\left[A_{i 1}^{\mathrm{w}}, \ldots, A_{i n}^{\mathrm{w}}\right]$, it follows that $A^{\mathrm{w}} \rightarrow B^{\mathrm{w}}$.

Proposition 4.8. Let $M \rightarrow N$ where root $(N)$ is white and every maximal special subterm with black root of $M$ is terminating. Then there exists a term $A$ such that $N \equiv_{0} A, A \rightarrow{ }_{i} N, M \rightarrow A$, and $M^{w} \rightarrow A^{w}$. (See diagram in Figure 13.) 

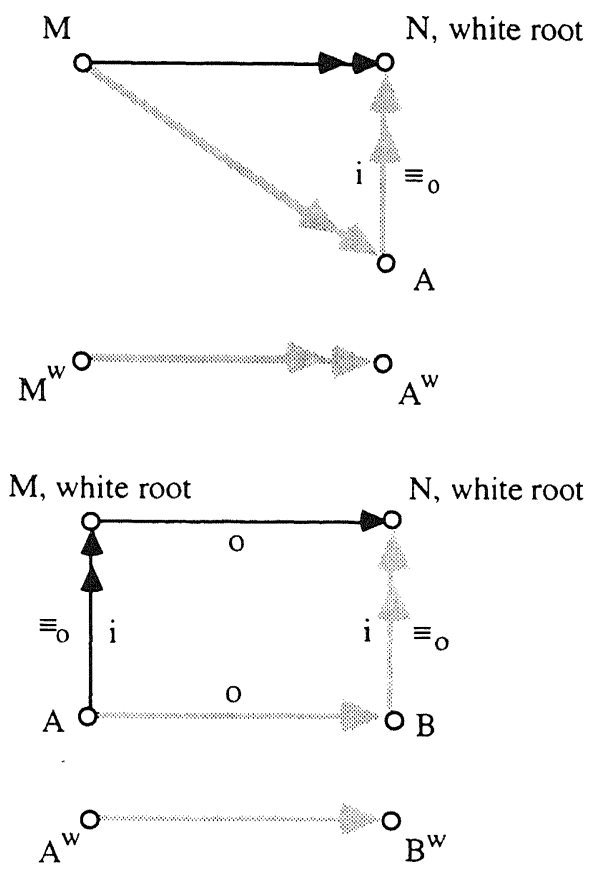

FIGURE 12

FIGURE 13

ProOF. We will prove the proposition by induction on $\operatorname{rank}(M)$.

Basis: $\operatorname{rank}(M)=1$. This case is trivial: Take $A \equiv N$.

Induction hypothesis: The proposition holds for $M$ with $\operatorname{rank}(M)<k$. Now let $M$ have rank $k$.

ClaIM. The proposition holds if $M \rightarrow_{i} N$.

ProOF OF THE ClaIM. Let $M \equiv C\left[M_{1}, \ldots, M_{m} \rrbracket \rightarrow_{i} N \equiv C\left[N_{1}, \ldots, N_{m}\right]\right.$ where $M_{i} \rightarrow N_{i}$ for $i=1, \ldots, m$. Without loss of generality we may assume that $N_{1} \equiv x, \ldots, N_{p-1} \equiv x$, $\operatorname{root}\left(N_{i}\right)$ is white for $p \leq i<q$, and $\operatorname{root}\left(N_{j}\right)$ is black for $q \leq j \leq m$. Thus,

$$
N \equiv C\left[x, \ldots, x, N_{p}, \ldots, N_{q-1}, N_{q}, \ldots, N_{m}\right] .
$$

By the induction hypothesis, for every $M_{i}(p \leq i<q)$ there is a term $A_{i}$ such that we have the diagram in figure 14 .

Now take $A \equiv C\left[x, \ldots, x, A_{p}, \ldots, A_{q-1}, M_{q}, \ldots, M_{m}\right]$. Clearly, $M \rightarrow A$. Since $A_{i} \equiv_{0} N_{i}(p \leq i<q)$ and both $M_{j}, N_{j}(q \leq j \leq m)$ have black root, we have $A \equiv_{0} N$. Furthermore, $A \rightarrow_{i} N$ since $A_{i} \rightarrow_{i} N_{i}(p \leq i<q)$ and by Proposition 3.4 the reductions $M_{j} \rightarrow N_{j}(q \leq j \leq m)$ can be taken such that every term in them has a black root. Now

$$
\begin{aligned}
M^{\mathrm{w}} & \equiv C\left[M_{1}^{\mathrm{w}}, \ldots, M_{p-1}^{\mathrm{w}}, M_{p}^{\mathrm{w}}, \ldots, M_{q-1}^{\mathrm{w}}, M_{q}^{\mathrm{w}}, \ldots, M_{m}^{\mathrm{w}}\right], \\
A^{\mathrm{w}} & \equiv C\left[x, \ldots, x, A_{p}^{\mathrm{w}}, \ldots, A_{q-1}^{\mathrm{w}}, M_{q}^{\mathrm{w}}, \ldots, M_{m}^{\mathrm{w}}\right],
\end{aligned}
$$

(for $A^{\mathrm{w}}$, see Remark 4.3). By Proposition 4.2(iv) we have $M_{i}^{\mathrm{w}} \rightarrow x(1 \leq i<p$ ), since $M_{i} \rightarrow x$. We had already $M_{i}^{\mathrm{w}} \rightarrow A_{i}^{\mathrm{w}}(p \leq i<q)$. Hence, $M^{\mathrm{w}} \rightarrow A^{\mathrm{w}}$. (See figure 15.) (End of the Claim) 
FIGURE 14

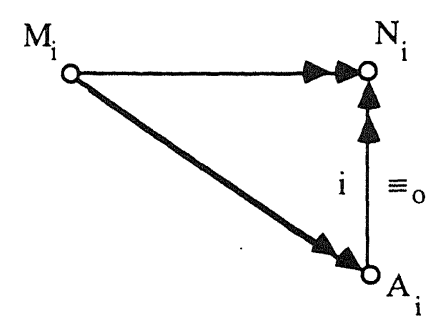

$(\mathrm{p} \leq \mathrm{i}<\mathrm{q})$


FIGURE 15

Now we will prove the full proposition (without the additional assumption $M \rightarrow_{i} N$ as in the Claim) for $\operatorname{rank}(M)=k$. We distinguish two cases.

Case 1. The root of $M$ is white.

So $M, N$ have both white roots. Hence, there is, by Proposition 3.4, a reduction $M \rightarrow N$ in which every term has white root. This reduction can be split into

$$
M \rightarrow_{\mathrm{i}} \rightarrow_{\mathrm{o}} \rightarrow_{\mathrm{i}} \rightarrow_{\mathrm{o}} \cdots \rightarrow_{\mathrm{i}} N .
$$

Now we can construct the diagram as in Figure 16.

Here subdiagrams $\alpha$ are justified by the Claim, subdiagrams $\beta$ by Proposition 4.7 and subdiagrams $\gamma$ follow by transitivity of $\rightarrow_{i}$. 


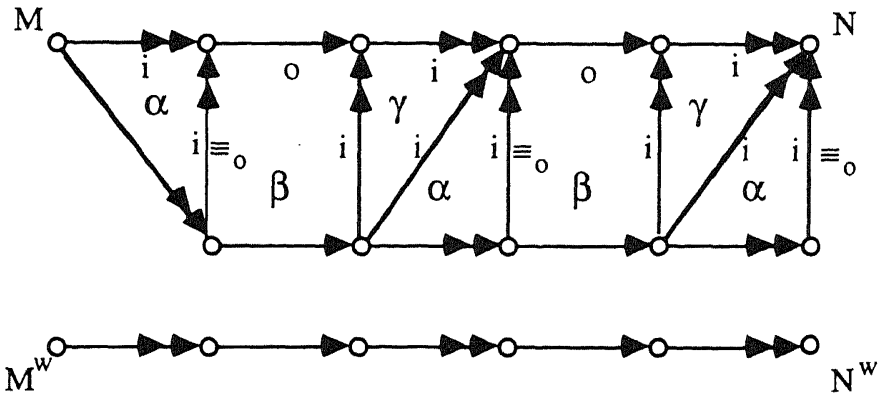

FIGURE 16

Case 2. The root of $M$ is black.

By Lemma 3.7.2, there is an essential subterm $Q$ of $M$ such that $M \rightarrow Q \rightarrow$ $N$. By Proposition 4.2(iii), $M^{\mathrm{w}} \rightarrow Q^{\mathrm{w}}$. Obviously, $\operatorname{rank}(Q)<\operatorname{rank}(M)=k$. Hence, we can construct the diagram in Figure 17, where the triangular subdiagram is obtained by the induction hypothesis applied on $Q$.

We are now able to state and prove the main result of our paper:

THEOREM 4.9. Let $R_{b}, R_{w}$ be left-linear and complete. Then $R_{b} \oplus R_{w}$ is a terminating TRS.

Proof. Let $M \in \operatorname{Ter}\left(R_{\mathrm{b}} \oplus R_{\mathrm{w}}\right)$. We will prove by induction on $\operatorname{rank}(M)$ that $M$ does not have an infinite reduction.

Without loss of generality, we may assume that $M$ has a white root. The case $\operatorname{rank}(M)=1$ is trivial, by assumption. Induction hypothesis: If $\operatorname{rank}(M)<k$, $M$ cannot have an infinite reduction. Now suppose for a proof by contradiction that there is a term $M$ with $\operatorname{rank}(M)=k$ having an infinite reduction $M \equiv M_{0} \rightarrow M_{1} \rightarrow M_{2} \rightarrow \cdots$. Now $\operatorname{rank}\left(M_{0}\right) \geq \operatorname{rank}\left(M_{1}\right) \geq \cdots ;$ by the induction hypothesis it follows that $\operatorname{rank}\left(M_{0}\right)=\operatorname{rank}\left(M_{1}\right)=\cdots$. Hence, the roots of all $M_{i}$ are white. Note that every principal subterm of $M_{i}$ is terminating.

Now infinitely many steps $M_{i} \rightarrow M_{i+1}$ must be in fact $M_{i} \rightarrow_{\mathrm{o}} M_{i+1}$; otherwise, we would have an infinite internal reduction

$$
M_{k} \equiv C_{k} \llbracket M_{k, 1}, \ldots, M_{k, r} \rrbracket \rightarrow_{\mathrm{i}} \rightarrow_{\mathrm{i}} \rightarrow_{\mathrm{i}} \cdots,
$$

which would yield an infinite reduction of some $M_{k, p}$, in contradiction with the induction hypothesis.

So, we can apply the following diagram construction (Figure 18), using Propositions 4.7 and 4.8 in the same way as for Figure 16. But this means that $M^{\mathrm{w}}$ has already an infinite reduction, in contradiction with the termination property of $R_{\mathrm{w}}$.

COROLlary 4.10. Let $R_{b}, R_{w}$ be left-linear. Then:

$$
R_{b} \oplus R_{w} \text { is complete } \Leftrightarrow R_{b} \text { and } R_{w} \text { are complete. }
$$

PROOF

$(\Rightarrow)$ is trivial.

$(\Leftarrow)$ follows from Theorem 4.9 and the theorem in Toyama [1987a] stating that for all TRSs, $R_{\mathrm{b}} \oplus R_{\mathrm{w}}$ is confluent iff $R_{\mathrm{b}}, R_{\mathrm{w}}$ are confluent. 
FIGURE 17
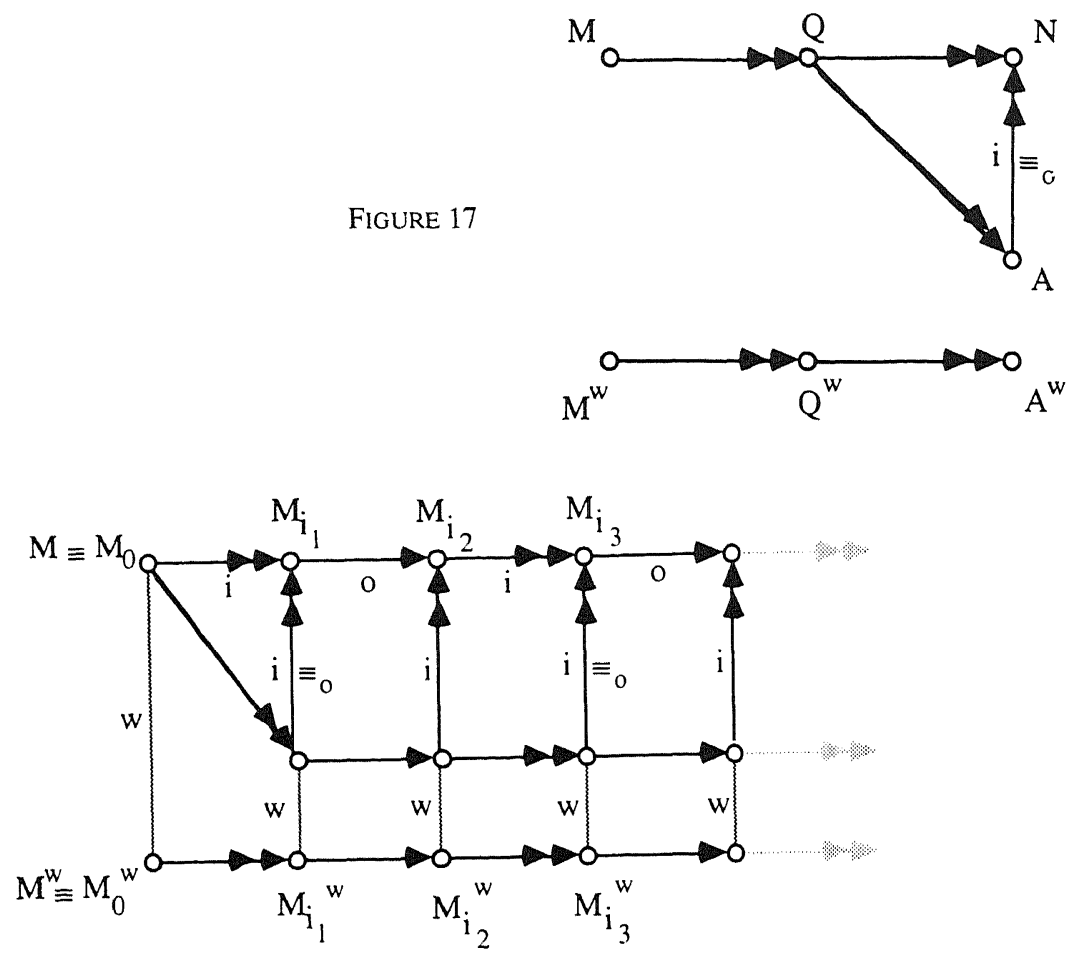

FIGURE 18

\section{Projection of mixed terms}

In this section, we will prove Proposition 4.2 for the direct sum $R_{\mathrm{b}} \oplus R_{\mathrm{w}}$ of left-linear and complete $R_{\mathrm{b}}, R_{\mathrm{w}}$, that is, the existence of the white (black) projection. To this end, we first define the white (black) projection and next prove that the defined projection satisfies the properties (i)-(iv) in Proposition 4.2.

The definition of the projections is rather subtle and rests heavily upon the Main Lemma 3.8.5. We will prepare the way by an example. Suppose $M$ is structured as in Figure 19(a); a concrete example is: $M \equiv F(g(C), h(C))$ as in Figure 19(b) where $R_{\mathrm{b}}=\{F(x, C) \rightarrow x, F(C, x) \rightarrow x\}$ and $R_{\mathrm{w}}=\{g(x) \rightarrow$ $x, h(x) \rightarrow x\}$. So $P_{1} \equiv g(C), P_{2} \equiv h(C)$ are the essential subterms of $M$. Now suppose we wish to determine the white projection $M^{\text {w }}$. As $M$ can collapse to $P_{1}$ as well as to $P_{2}$, the projection $M^{\mathrm{w}}$ should convey the information in both $P_{1}, P_{2}$. The problem is that these subterms are disjoint (in this case). Yet, there is a way to combine them into one term: namely by piling them with result as in Figure 19(c), respectively 19(d). Throughout this section, the variable $x$ will play a special role.

Of course, we were lucky in this example, since the white top triangles of $P_{1}$, $P_{2}$ which we wanted to pile, were indeed "pileable." In the situation of Figure 20 , where $P_{1}$ is supposed to be again nondeterministic, the piling would not have succeeded, because triangles 1,2 can be taken such that they cannot be piled. However, our Main Lemma 3.8.5 says that such a situation does not exist and, therefore, piling succeeds as will be proved in more detail below. 


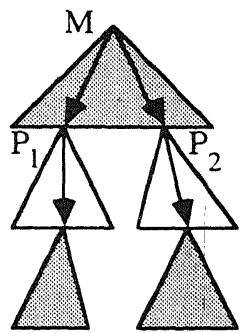

(a)

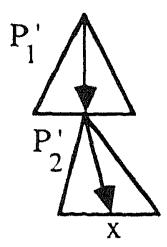

(c)

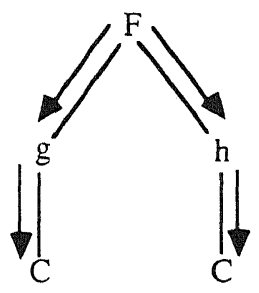

(b)

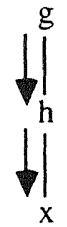

(d)

FIGURE 19

Definition 5.1. Let $R$ be a confluent and left-linear TRS. Let $P_{1}, \ldots, P_{p}$ be a sequence of terms of $R(p \geq 2)$. Then, the term pile $\left(P_{1}, \ldots, P_{p}\right)$ is defined as follows:

Case 1. $P_{i} \rightarrow x$ for $i=1, \ldots, p$. So $P_{i} \equiv C_{i}[x]$ such that $C_{i}[\underline{x}] \rightarrow \underline{\mathrm{x}}$ (there may be other occurrences of not underlined $x$ 's in $\left.C_{i}[\mathrm{x}]\right)$.

Then pile $\left(P_{1}, \ldots, P_{p}\right) \equiv C_{1}\left[C_{2}\left[\cdots C_{p-1}\left[C_{p}[x]\right] \cdots\right]\right]$.

Case 2. Not case 1: Then pile $\left(P_{1}, \ldots, P_{p}\right)$ is undefined.

Example 5.1.1. Note that pile $\left(P_{1}, \ldots, P_{p}\right)$ does not merely depend on $P_{1}, \ldots, P_{p}$ but also on $R$. If $R=\{F(x, y) \stackrel{p}{\rightarrow} x, I(x) \rightarrow x\}$ and $P_{1} \equiv F(x, x)$, $P_{2} \equiv I(x)$, then pile $\left(P_{1}, P_{2}\right) \equiv F(I(x), x)$. If in $R$ the first rule is replaced by $F(x, y) \rightarrow y$, then $\operatorname{pile}\left(P_{1}, P_{2}\right) \equiv F(x, I(x))$.

Remark 5.1.2. The condition in Definition 5.1, that $R$ is confluent and left-linear, is necessary to ensure that pile is a (partial) function. Otherwise, taking $R=\{F(x, y) \rightarrow x, F(x, y) \rightarrow y, I(x) \rightarrow x\}$ and $P_{1} \equiv F(x, x), P_{2} \equiv I(x)$, we would have (see the previous example) pile $\left(P_{1}, P_{2}\right) \equiv F(x, I(x))$ as well as $F(I(x), x)$. That confluence and left-linearity of $R$ is sufficient to make pile into a function, is easily seen as follows. Now assume that $C[\underline{x}, x] \rightarrow \underline{x}$ as well as $C[x, \underline{x}] \rightarrow \underline{x}$. Then this implies (by left-linearity) that $C[x, y] \rightarrow x$ as well as $y$, contradicting confluence.

In the sequel, we will use pile for terms of $R_{\mathrm{b}} \oplus R_{\mathrm{w}}$, where $R_{\mathrm{b}}, R_{\mathrm{w}}$ are complete and left-linear. Indeed, the direct sum is then confluent (and, trivially, left-linear), as guaranteed by the theorem in Toyama [1987] stating that the direct sum of confluent TRSs is again confluent. Thus, the operation pile is well defined. 
FIGURE 20

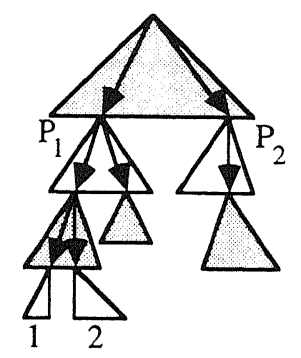

Definition 5.2. Let every maximal special subterm with black (white) root of $M \in \operatorname{Ter}\left(R_{0} \oplus R_{1}\right)$ be terminating (if it exists). Then, the white projection $M^{\mathrm{w}}$ of $M$ is inductively defined as follows:

(1) If $M \equiv x$, then $M^{\mathrm{w}} \equiv x$.

(2) $M \equiv f\left(M_{1}, \ldots, M_{n}\right)(n \geq 0)$ has a white root. Then, $M^{\mathrm{w}} \equiv f\left(M_{1}^{\mathrm{w}}, \ldots, M_{n}^{\mathrm{w}}\right)$.

(3) $M$ has a black root:

(3.1) $M$ has no essential subterm. Then, $M^{\mathrm{w}} \equiv x$.

(3.2) $M$ has precisely one essential subterm $P$. Then, $M^{\mathrm{w}} \equiv P^{\mathrm{w}}$.

(3.3) $M$ is mixed nondeterministic, with sequence of essential subterms $P_{1}, \ldots, P_{p}$. Then, $M^{\mathrm{w}} \equiv \operatorname{pile}\left(P_{1}^{\mathrm{w}}, \ldots, P_{p}^{\mathrm{w}}\right)$.

(The black projection $M^{\mathrm{b}}$ is defined by interchanging $\mathrm{b}, \mathrm{w}$ and black, white.) In case (3.3), the essential subterm occurrences $P_{1}, \ldots, P_{p}$ may be ordered by precedence of their head symbol. (The precise ordering is irrelevant.) Note that $M^{\mathrm{w}}$ may be undefined, due to the possible undefinedness of $\operatorname{pile}\left(P_{1}^{\mathrm{w}}, \ldots, P_{p}^{\mathrm{w}}\right)$. Lemma 5.7 will however show that in the present situation, where $R_{\mathrm{b}}, R_{\mathrm{w}}$ are left-linear and complete, if every maximal special subterm with black (white) root of $M$ is terminating then $M^{\mathrm{w}}\left(M^{\mathrm{b}}\right)$ is well defined. Note that (3.2) is not a special case of (3.3) since in general $\operatorname{pile}(N) \not \equiv N$, even if the definition of pile $\left(P_{1}, \ldots, P_{p}\right)(p \geq 2)$ is extended to that of pile $\left(P_{1}, \ldots, P_{p}\right)(p \geq 1)$. (In fact: $\operatorname{pile}(N) \equiv N \Leftrightarrow \operatorname{pile}(N)$ is defined $\Leftrightarrow N \rightarrow x$.) Finally, note that in (3.2), (3.3) we have $\operatorname{rank}(P)<\operatorname{rank}(M)$ and $\operatorname{rar}_{-}^{1}\left(P_{i}\right)<\operatorname{rank}(M)$, respectively.

Example 5.3. (See Figure 21.)

Example 5.4. (See Figure 22.)

Example 5.5. Consider the TRSs $R_{\mathrm{b}}=\{F(C(y), x) \rightarrow x, F(x, C(y)) \rightarrow$ $x, C(y) \rightarrow D\}, R_{\mathrm{w}}=\{g(x) \rightarrow x, h(x) \rightarrow x\}$ with $R_{\mathrm{w}}$ containing also a constant ' $a$ '. Then

$$
\begin{aligned}
(F(g(C(a)), h(C(a))))^{\mathrm{w}} & =\operatorname{pile}\left((g(C(a)))^{\mathrm{w}},(h(C(a)))^{\mathrm{w}}\right) \\
& =\operatorname{pile}\left(g\left((C(a))^{\mathrm{w}}\right), h\left((C(a))^{\mathrm{w}}\right)\right) \\
& =\operatorname{pile}(g(x), h(x))=g(h(x)) .
\end{aligned}
$$

Example 5.6. The black projection of the following term (in Figure 23) is undefined; however, by the Main Lemma (3.8.5) such terms cannot exist (when $R_{\mathrm{b}}, R_{\mathrm{w}}$ are left-linear and complete and every maximal special subterm with white root of $M$ is terminating). 
Direct Sums of Left-Linear Complete Term Rewriting Systems
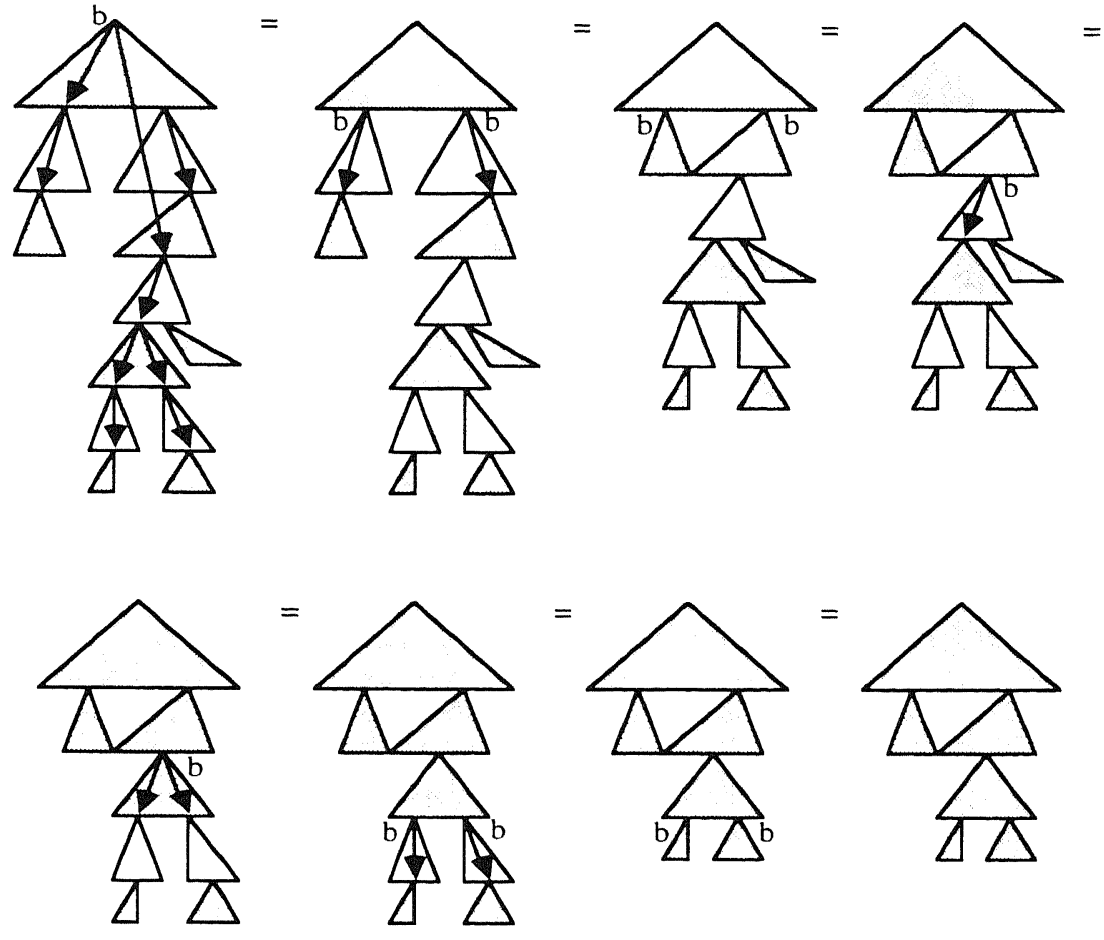

FIGURE 21
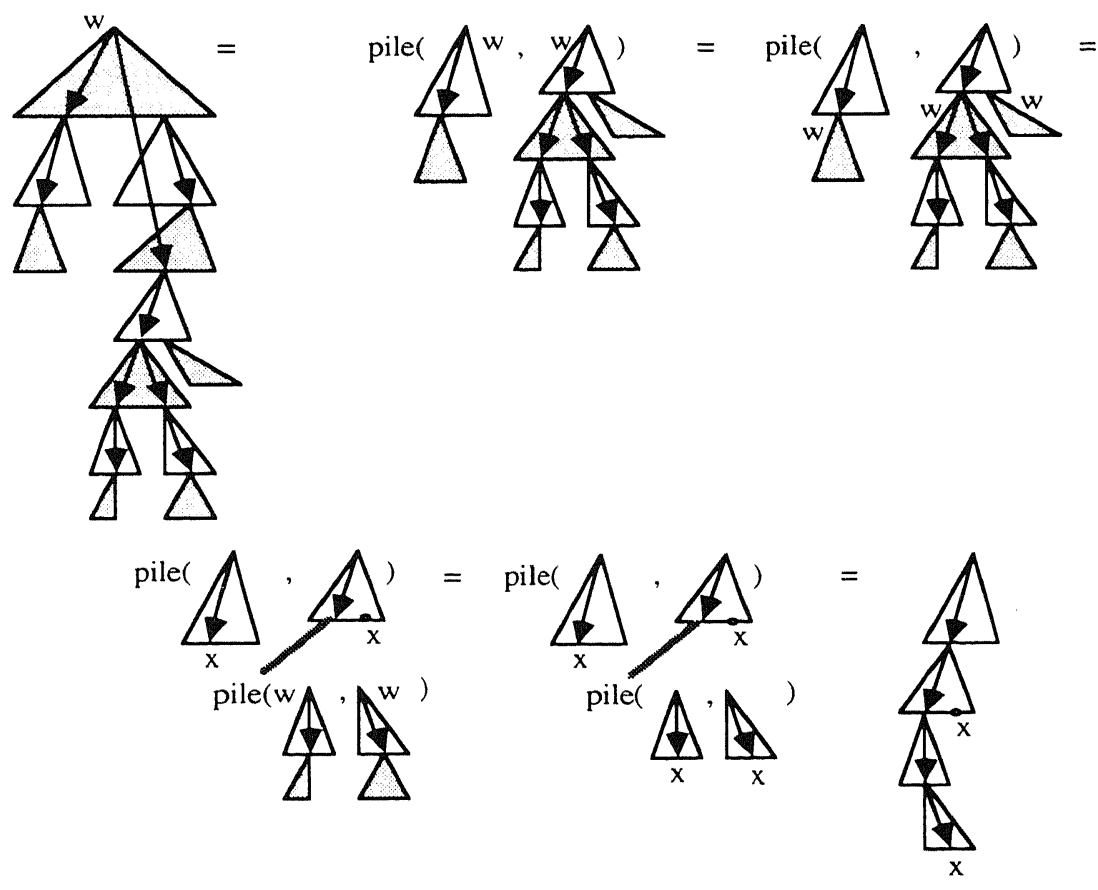

FIGURE 22 



FIGURE 23

In many cases, the result of projecting $M$ to $M^{\mathrm{b}}$ or $M^{\mathrm{w}}$ will be a term collapsing to the special variable $x$ (i.e. $M^{\mathrm{b}} \rightarrow x$; respectivey, $M^{\mathrm{w}} \rightarrow x$.) See for example, Example 5.6. We will now prove simultaneously this fact and the well-definedness of $M^{\mathrm{w}}\left(M^{\mathrm{b}}\right)$ when every maximal special subterm with black (white) root of $M$ is terminating.

LEMMA 5.7. Let every maximal special subterm with black (white) root of $M \in \operatorname{Ter}\left(R_{b} \oplus R_{w}\right)$ be terminating (if it exists). Then the white projection $M^{w}$ (the black projection $M^{b}$ ) of $M$ is well defined and $M^{w} \rightarrow x \Leftrightarrow \operatorname{root}(M \downarrow) \notin \mathscr{F}_{w}$ (respectively, $M^{b} \rightarrow x \Leftrightarrow \operatorname{root}(M \downarrow) \notin \mathscr{F}_{b}$ ).

ProOF. We will prove a slightly stronger statement, namely (i) and (ii):

(i) If $\operatorname{root}(M \downarrow) \notin \mathscr{F}_{\mathrm{w}}$, then $M^{\mathrm{w}}$ is well-defined and $M^{\mathrm{w}} \rightarrow x$,

(ii) If $\operatorname{root}(M \downarrow) \in \mathscr{F}_{\mathrm{w}}$ and $M \downarrow \equiv C^{*} \llbracket M_{1}, \ldots, M_{m} \rrbracket(m \geq 0)$, then $M^{\mathrm{w}}$ is well-defined and $M^{\mathrm{w}} \downarrow \equiv C^{*}[x, \ldots, x]$. (Hence: Not $M^{\mathrm{w}} \rightarrow x$.)

We will prove (i) and (ii) by induction on $\operatorname{rank}(M)$.

Basis. $\operatorname{rank}(M)=1$.

Case 1. $M \in \operatorname{Ter}\left(R_{w}\right)$. Then $M^{\mathrm{w}} \equiv M$, by (1) or (2) of Definition 5.2. If $M \downarrow \equiv x$, then $M^{\mathrm{w}} \equiv M \rightarrow x$, so (i) Ł.Jlds; (ii) holds vacuously. If $\operatorname{root}(M \downarrow) \in$ $\mathscr{F}_{\mathrm{w}}$, then (i) holds vacuously; (ii) holds since $M^{\mathrm{w}} \downarrow \equiv M \downarrow$.

Case 2. $M \in \operatorname{Ter}\left(R_{\mathrm{b}}\right)$. We may suppose $M \not \equiv x$, since the case $M \equiv x$ was covered in case 1. By (3.1) of Definition 5.2, $M^{\mathrm{w}} \equiv x$. So (i) holds. Statement (ii) holds vacuously.

Induction hypothesis. Assume (i) and (ii) hold for $\operatorname{rank}(M)<k(k \geq 2)$.

Now consider $M$ with $\operatorname{rank}(M)=k$.

Case 1. $\operatorname{root}(M) \in \mathscr{F}_{w}$. Let $M \equiv C \llbracket M_{1}, \ldots, M_{m} \rrbracket(m \geq 1)$, so $M^{\mathrm{w}} \equiv$ $C\left[M_{1}^{\mathrm{w}}, \ldots, M_{m}^{\mathrm{w}}\right]$. Without loss of generality, we may assume that $\operatorname{root}\left(M_{i} \downarrow\right) \notin$ $\mathscr{F}_{\mathrm{w}}$ for $1 \leq i<p$ and $\operatorname{root}\left(M_{j} \downarrow\right) \in \mathscr{F}_{\mathrm{w}}$ for $p \leq j \leq m$. So, by the induction hypothesis: every $M_{i}^{\mathrm{w}}$ is well-defined, $M_{i}^{\mathrm{w}} \rightarrow x(1 \leq i<p)$, and writing $M_{j} \downarrow$ $\equiv C_{j}^{*}\left[N_{j, 1}, \ldots, N_{j, n j} \rrbracket\left(n_{j} \geq 0, p \leq j \leq m\right): M_{j}^{\mathrm{w}} \downarrow \equiv C_{j}^{*}[x, \ldots, x]\right.$. Thus

$M \downarrow \equiv C\left[M_{1} \downarrow, \ldots, M_{m} \downarrow\right] \downarrow$

$$
\equiv C\left[M_{1} \downarrow, \ldots, M_{p-1} \downarrow, C_{p}^{*} \llbracket N_{p, 1}, \ldots, N_{p, n p} \rrbracket, \ldots, C_{m}^{*} \llbracket\left[N_{m, 1}, \ldots, N_{m, n m} \rrbracket\right] \downarrow\right.
$$


and

$$
\begin{aligned}
M^{\mathrm{w}} \downarrow & \equiv C\left[M_{1}^{\mathrm{w}} \downarrow, \ldots, M_{m}^{\mathrm{w}} \downarrow\right] \downarrow \\
& \equiv C\left[x, \ldots, x, C_{p}^{*}[x, \ldots, x], \ldots, C_{m}^{*}[x, \ldots, x]\right] \downarrow .
\end{aligned}
$$

Note that $M_{1} \downarrow, \ldots, M_{p-1} \downarrow, N_{p, 1}, \ldots, N_{m, n m}$ are normal forms having roots not in $\mathscr{F}_{\mathrm{w}}$. Therefore, if $\operatorname{root}(M \downarrow) \notin \mathscr{F}_{\mathrm{w}}$, then

$$
C\left[x, \ldots, x, C_{p}^{*}[x, \ldots, x], \ldots, C_{m}^{*}[x, \ldots, x]\right] \downarrow \equiv x
$$

and if $\operatorname{root}(M \downarrow) \in \mathscr{F}_{\mathrm{w}}$, then we have a context $C^{*}[, \ldots,] \equiv$ $C\left[, \ldots, C_{p}^{*}[, \ldots],, \ldots, C_{m}^{*}[, \ldots],\right] \downarrow$ such that $M \downarrow \equiv C^{*}\left[N_{1}, \ldots, N_{n}\right]$ where $N_{i}$ $\in\left\{M_{1} \downarrow, \ldots, M_{p-1} \downarrow, N_{p, 1}, \ldots, N_{m, n m}\right\}$ and $M^{\mathrm{w}} \downarrow \equiv C^{*}[x, \ldots, x] \not \equiv x$ (using $N_{p, 1}^{\mathrm{w}} \equiv \cdots \equiv N_{n, n m}^{\mathrm{w}} \equiv x$ by (3.1) of Definition 5.2).

Case 2. $\operatorname{root}(M) \notin \mathscr{F}_{\mathrm{w}}$. Distinguish the subcases:

Case 2.1. $M$ has no essential subterm. Then, $M^{\mathrm{w}} \equiv x$, either by (1) of Definition 5.2 or (3.1). Hence, $M^{\mathrm{w}} \downarrow \equiv x$, and (i) and (ii) hold.

Case 2.2. $M$ has precisely one essential subterm $P$. Then $M^{\mathrm{w}} \equiv P^{\mathrm{w}}$. Note that $\operatorname{rank}(P)<k$. Thus, by the induction hypothesis every $P^{\mathrm{w}}$ is well defined. Since $M \downarrow \equiv P \downarrow$ and $M^{\mathrm{w}} \downarrow \equiv P^{\mathrm{w}} \downarrow$, the claim follows by using the induction hypothesis.

Case 2.3. $M$ has essential subterms $P_{1}, \ldots, P_{p}(p>1)$. Note that $\operatorname{rank}\left(P_{i}\right)$ $<k$ for all $i$. By the Main Lemma 3.8.5, $\operatorname{root}(M \downarrow) \notin \mathscr{F}_{\mathrm{w}}$. Since $M \downarrow \equiv P_{i} \downarrow$, also $\operatorname{root}\left(P_{i} \downarrow\right) \notin \mathscr{F}_{\mathrm{w}}$ for all $i$. So, by the induction hypothesis, $P_{i}^{\mathrm{w}} \rightarrow x$ for all $i$. Now $M^{\mathrm{w}} \equiv \operatorname{pile}\left(P_{1}^{\mathrm{w}}, \ldots, P_{p}^{\mathrm{w}}\right)$ and since $P_{i}^{\mathrm{w}} \rightarrow x(i=1, \ldots, p), M^{\mathrm{w}}$ is defined. Obviously, $M^{\mathrm{w}} \equiv \operatorname{pile}\left(P_{1}^{\mathrm{w}}, \ldots, P_{p}^{\mathrm{w}}\right) \rightarrow x$. Hence, (i) is true and (ii) holds vacuously.

Proposition 5.8. Let $M$ have a black root and let $M$ be terminating. Suppose $P$ is an essential subterm of $M$. Then $M^{w} \rightarrow P^{w}$.

Proof. See Definition 5.2 of $M^{\mathrm{w}}$. The only possible cases are (3.2) and (3.3). In case (3.2), $M^{\mathrm{w}} \equiv P^{\mathrm{w}}$. In case (3.3), $M^{\mathrm{w}} \equiv \operatorname{pile}\left(P_{1}^{\mathrm{w}}, \ldots, P_{p}^{\mathrm{w}}\right)$ where $P \equiv P_{k}$ for some $k \in\{1, \ldots, p\}$. From Main Lemma 3.8.5, $M$ cannot have color change, that is, $\operatorname{root}(M \downarrow)$ is black or $M \downarrow \equiv x$. By $M \downarrow \equiv P_{1} \downarrow \equiv \cdots \equiv P_{p} \downarrow$ and Lemma 5.7, $P_{i}^{\mathrm{w}} \rightarrow x(i=1, \ldots, p)$. Thus, we can write that $P_{i}^{\mathrm{w}} \equiv C_{i}[x]$ such that $C_{i}[\underline{z}] \rightarrow \underline{z}$. Hence, by definition of "pile":

$$
M^{\mathrm{w}} \equiv C_{1}\left[\cdots\left[C_{p}[x]\right] \cdots\right],
$$

which yields $M^{\mathrm{w}} \rightarrow C_{k}[x] \equiv P_{k}^{\mathrm{w}}$.

Proposition 5.9 (Proposition 4.2). Let every maximal special subterm with black (white) root of $M \in \operatorname{Ter}\left(R_{b} \oplus R_{w}\right)$ be terminating (if it exists). Then the white projection $M^{w}$ (the back projection $M^{b}$ ) of $M$ has the properties (where the black projection $M^{b}$ has the properties obtained by interchanging $w, b$ and white and black.):

(i) If $M \equiv x$ then $M^{w} \equiv x$.

(ii) If $M \equiv f\left(M_{1}, \ldots, M_{n}\right)(n \geq 0)$ has a white root, then $M^{w} \equiv f\left(M_{1}^{w}, \ldots, M_{n}^{w}\right)$.

(iii) If $M$ has a black root and an essential subterm $N$, then $M^{w} \rightarrow N^{w}$.

(iv) If $M \rightarrow x$ then $M^{w} \rightarrow x$. 
Proof. The proposition follows from Definition 5.2, Lemma 5.7, and Proposition 5.8.

\section{Appendix $A$}

In this appendix, we prove Lemma 3.8.5 (Main Lemma) in Section 3:

Lemma 3.8.5. Main Lemma. Let $R_{b}, R_{w}$ be left-linear and complete. Let $M$ be terminating and let $M$ have color change. Then $M$ has exactly one essential subterm.

Proof. First, we note that from color change of $M$ and Lemma 3.6, $M$ must have at least one essential subterm. Let $\mathbf{P}(M)$ be a predicate that if $M$ is terminating and has color change then $M$ has exactly one essential subterm. We prove $\forall M \mathbf{P}(M)$ by noetherian induction (see Huet [1980]), showing the claim:

$$
\forall M[\forall N[M \rightarrow N \Rightarrow \mathbf{P}(N)] \Rightarrow \mathbf{P}(M)] .
$$

For a proof of this claim by contradiction, suppose that there exists a terminating term $M$ such that $M$ has color change but more than one essential subterm and every $N$ obtained from $M$ with one step reduction (i.e., $M \rightarrow N$ ) satisfies $\mathbf{P}(N)$. Without loss of generality, we may suppose that $M$ has a black root.

Let $N$ be a term obtained from $M$ with one step reduction. If $N$ has a white root, then we can write $M \equiv C \llbracket M_{1}, \ldots, M_{k}, N, M_{k+1}, \ldots, M_{m} \rrbracket \rightarrow N$. From Proposition 2.9, $N$ must contain all the essential subterms of $M$. In particular, $N$ must contain an essential subterm $P$ of $M$ as a proper subterm, since $M$ has more than one essential subterm. But this contradicts the fact that $N$ is also an essential subterm of $M$. Thus, $N$ must have a black root. From $M \downarrow \equiv N \downarrow, N$ has color change. $N$ is clearly terminating. Hence, $N$ must have exactly one essential subterm since $\mathbf{P}(N)$ holds.

Let $P$ and $Q$ be two essential subterms of $M$. Let $M_{P}$ ( $M_{Q}$, respectively) be the minimal special subterm occurrence with black root that contains $P(Q$ respectively). We have to show that all the possible positions of $M_{P}$ and $M_{Q}$ in $M$ contradict the uniqueness of the essential subterm of $N$.

Case 1. $M_{P}$ and $M_{Q}$ coincide.

Let $M \equiv C\left[M_{P}\right]\left(\equiv C\left[M_{Q}\right]\right)$. Since $M_{P}$ is not only the minimal special subterm occurrence with black root containing $P$ but also that containing $Q, P$ and $Q$ must occur independently in $M_{P}$. Thus, we can write $M_{P} \equiv C_{P}[P, Q]$.

If $C[z]$ is not in normal form, we have a reduction $C[z] \rightarrow C^{\prime}[z, \ldots, z]$. By Proposition 2.9, $C^{\prime}[z, \ldots, z]$ must contain at least one occurrence of $z$. Thus, $N \equiv C^{\prime}\left[M_{P}, \ldots, M_{P}\right]$ keeps two essential subterms $P$ and $Q$; this contradicts the fact that $N$ must have exactly one essential subterm. Hence, $C[z]$ must be a normal form.

Assume that $C_{P}\left[z, z^{\prime}\right]$ is a normal form. Then $C\left[C_{P}[P \downarrow, Q \downarrow]\right]$ is a normal form since $C\left[C_{P}\left[z, z^{\prime}\right]\right]$ is a normal form and $P \downarrow$ and $Q \downarrow$ have white roots. However, this contradicts color change of $M$. Thus, $C_{P}\left[z, z^{\prime}\right]$ must be not in normal form. We can write $C_{P}\left[z, z^{\prime}\right] \rightarrow C_{P}^{\prime}\left[z, \ldots, z, z^{\prime}, \ldots, z^{\prime}\right]$. By Proposition 2.9, $C_{p}^{\prime}\left[z, \ldots, z, z^{\prime}, \ldots, z^{\prime}\right]$ must contain the occurrences of $z$ and $z^{\prime}$. Thus, we 
have $N \equiv C\left[C^{\prime}{ }_{p}[P, \ldots, P, Q, \ldots, Q]\right]$ in which $P$ and $Q$ are two essential subterms of $N$ : contradiction. Thus, Case 1 is impossible.

Case 2. $M_{Q} \subset M_{P}$ (or $\left.M_{P} \subset M_{Q}\right)$.

Case 2.1. $M_{Q} \not \subset P$.

Let $M \equiv C\left[M_{P}\right], M_{P} \equiv C_{P}\left[P, M_{Q}\right]$ and $M_{Q} \equiv C_{Q}[Q]$. From a similar reason as for Case 1 , it follows that $C[z]$ and $C_{P}\left[z, z^{\prime}\right]$ must be normal forms. If $C_{Q}[z]$ is a normal form, then $C\left[C_{P}\left[P \downarrow, C_{Q}[Q \downarrow]\right]\right]$ must be a normal form of $M$ : contradiction to color change of $M$. Thus, $C_{O}[z]$ is not a normal form. Let $C_{Q}[z] \rightarrow C_{Q}^{\prime}[z, \ldots, z]$. By Proposition $2.9, C_{Q}^{\prime}[z, \ldots, z]$ contains at least one occurrence of $z$. If $C_{Q}^{\prime}[z, \ldots, z] \not \equiv z$, then we have $M \equiv C\left[C_{P}\left[P, C_{Q}[Q]\right]\right] \rightarrow$ $C\left[C_{P}\left[P, C_{Q}^{\prime}[Q, \ldots, Q]\right]\right]$ where $N$ has two essential subterms $P$ and $Q$ : contradiction. Thus, $C_{Q}[z]$ must collapse to $z$, that is, $C_{Q}[z] \rightarrow z$. Thus, we have $M \equiv C\left[C_{P}\left[P, C_{Q}[\underline{Q}]\right]\right] \rightarrow C\left[C_{P}[P \downarrow, Q]\right]$. However, since $C\left[C_{P}[P \downarrow, z]\right]$ is normal form, we cannot obtain a reduction $C\left[C_{P}[P \downarrow, Q]\right] \rightarrow Q$ without erasing the underlining of $Q$. This contradicts the fact that $\bar{Q}$ is an essential subterm of $M$. Hence, Case 2.1 is impossible.

Case 2.2. $M_{Q} \subset P$ (i.e., $Q \subset P$ ).

Let $M \equiv C[P]$. Then, from a similar reason as for Case 1 , it follows that $C[z]$ must be a normal form. Since $P \downarrow$ has a white root, $M \downarrow \equiv C[P \downarrow]$. This contradicts the color change of $M$; hence, Case 2.2 is impossible.

Case 3. $M_{P}$ and $M_{Q}$ occur independently (i.e., $M_{P} \nsubseteq M_{Q}$, and $M_{Q} \nsubseteq M_{P}$ ).

Let $M \equiv C\left[M_{P}, M_{Q}\right], M_{P} \equiv C_{P}[P]$, and $M_{Q} \equiv C_{Q}[Q]$. By a similar reason as for Case $1, C\left[z, z^{\prime}\right]$ must be a normal form. If $C_{P}[z]$ and $C_{Q}[z]$ both are normal forms, then we have $M \downarrow \equiv C\left[C_{P}[P \downarrow], C_{Q}[Q \downarrow]\right]$ : contradiction to the color change of $M$. Thus, both $C_{P}[z]$ and $C_{Q}[z]$ cannot be normal forms. Now consider the following two subcases:

Case 3.1. $C_{P}[z]$ is a normal form, but $C_{Q}[z]$ is not (or $C_{Q}[z]$ is a normal form but $C_{P}[z]$ is not).

Let $C_{Q}[z] \rightarrow C_{Q}^{\prime}[z, \ldots, z]$. If $C_{Q}^{\prime}[z, \ldots, z] \not \equiv z$, then $N \equiv$ $C\left[C_{P}\left[P, C_{Q}^{\prime}[Q, \ldots, Q]\right]\right]$ has two essential subterms $P$ and $Q$ by Proposition 2.9: contradiction. Thus, $C_{Q}[z] \rightarrow z$, and we have $M \equiv C\left[C_{P}\left[P, C_{Q}[Q]\right]\right] \rightarrow$ $C\left[C_{P}[P \downarrow, Q]\right]$. However, since $C\left[C_{P}[P \downarrow, z]\right.$ is a normal form, we cannot have $C\left[C_{P}[P \downarrow, \bar{Q}]\right] \rightarrow Q$. This contradicts the fact that $Q$ is an essential subterm of $M$. Hence, Case 3.1 is impossible.

Case 3.2. Neither $C_{P}[z]$ nor $C_{Q}[z]$ is a normal form.

By the same reason as for Case 3.1, we have $C_{P}[z] \rightarrow z$ and $C_{Q}[z] \rightarrow z$. Thus, we can write $M \equiv C\left[C_{P}[P], C_{Q}[Q]\right] \rightarrow N \equiv C\left[P, C_{Q}[Q]\right] \rightarrow N^{\prime} \equiv$ $C[P, Q]$. Note that since $N$ has exactly one essential subterm, by Proposition $2.9 Q$ must be this unique essential subterm of $N$. Since $N^{\prime}$ has a black root and $N^{\prime} \downarrow(\equiv M \downarrow)$ has a white root, $N^{\prime}$ must have at least one essential subterm, say $P^{\prime}$, by Lemma 3.7.2. However, we cannot take $P$ nor $Q$ as $P^{\prime}$ since $P$ and $Q$ are not special subterms of $N^{\prime}$. Which special subterm of $N^{\prime}$ can we take as $P^{\prime}$ ? If $P^{\prime}$ and $Q$ occur independently in $N^{\prime}$, we can write $N \equiv C^{\prime}\left[P^{\prime}, C_{Q}[Q]\right] \rightarrow N^{\prime} \equiv C^{\prime}\left[P^{\prime}, Q\right] \rightarrow P^{\prime}$. Hence, not only $Q$ but also $P^{\prime}$ is 
an essential subterm of $N$ : contradiction to the uniqueness of essential subterm of $N$. If $P^{\prime} \subset Q$, by a similar way we can easily show that $P^{\prime}$ is an essential subterm of $N$ : again contradiction. If $Q \subset P^{\prime}$, by Lemma 2.8 we have some essential subterm $P^{\prime \prime}$ in $N$ such that $P^{\prime \prime} \rightarrow P^{\prime}$ and $Q \subset P^{\prime \prime}$ : contradiction. Therefore, it follows that Case 3.2 is impossible.

\section{Appendix $B$}

In this Appendix we prove termination of the direct sum of a left-linear complete TRS and a non-collapsing terminating TRS. A TRS is noncollapsing if it does not contain collapsing rewrite rules; a rewrite rule is a collapsing rule if its right-hand side is a variable.

Throughout this Appendix, let $R_{\mathrm{b}}$ and $R_{\mathrm{w}}$ be terminating TRSs. Rusinowitch [1987] proved:

THEOREM B.1. If $R_{b}$ and $R_{w}$ are noncollapsing, then the direct sum $R_{b} \oplus R_{w}$ is terminating.

In the present paper, we have shown:

THEOREM B.2. If $R_{b}$ and $R_{w}$ are left-linear and confluent, then the direct sum $R_{b} \oplus R_{w}$ is terminating.

These two facts suggest that the properties "noncollapsing" and "left-linear and confluent" are in some instances interchangeable. Thus, we are led to the following conjecture.

CONJECTURE B.3. If $R_{b}$ is noncollapsing and $R_{w}$ is left-linear and confluent, then the direct sum $R_{b} \oplus R_{w}$ is terminating.

We will show that this conjecture indeed holds. Henceforth, we assume that $\mathrm{R}_{\mathrm{b}}$ is noncollapsing and terminating, and that $R_{\mathrm{w}}$ is left-linear and complete (i.e., confluent and terminating). Further, $\rightarrow_{\mathrm{i}}(i=\mathrm{b}, \mathrm{w})$ denotes the reduction relation given by the rewrite rules of $R_{i}$. Note that a mixed term $t$ has a unique normal form with respect to $\rightarrow_{\mathrm{w}}$, denoted by $n f_{\mathrm{w}}(t)$, because $R_{\mathrm{w}}$ is complete.

Lemma B.4. Let $t \rightarrow_{b}$ s. Then we have $n f_{w}(t) \rightarrow{ }_{b} n f_{w}(s)$. Moreover, if $t \rightarrow_{b} s$ is an outer reduction, then $n f_{w}(t) \rightarrow_{b} n f_{w}(s)$.

Proof. Since $t \rightarrow_{\mathrm{b}} s$, there exists a rule $1 \rightarrow r$ of $R_{\mathrm{b}}$ with the set of variables $\left\{x_{1}, \ldots, x_{n}\right\}$ occurring in 1 , a context $C[]$, and mixed terms $t_{1}, \ldots, t_{n}$, such that $t \equiv C[1 \sigma] \rightarrow_{\mathrm{b}} s \equiv C[\mathrm{r} \sigma]$, where the substitution $\sigma$ is defined by

$$
x_{i} \sigma \equiv t_{i}(i=1, \ldots, n) .
$$

Let $n f_{\mathrm{w}}(C[]) \equiv C^{\prime}[, \ldots$,$] . By x_{i} \sigma^{\prime} \equiv n f_{\mathrm{w}}\left(t_{i}\right)(i=1, \ldots, n)$ we define a new substitution $\sigma^{\prime}$. From the noncollapsing property $R_{\mathrm{b}}$ of and the left-linearity of $R_{\mathrm{w}}$, it follows that $n f_{\mathrm{w}}(t) \equiv C^{\prime}\left[1 \sigma^{\prime}, \ldots, 1 \sigma^{\prime}\right]$ and $n f_{\mathrm{w}}(s) \equiv C^{\prime}\left[\mathrm{r} \sigma^{\prime}, \ldots, \mathrm{r} \sigma^{\prime}\right]$. Thus, $n f_{\mathrm{w}}(t) \rightarrow_{\mathrm{b}} n f_{\mathrm{w}}(s)$ holds. If $t \rightarrow_{\mathrm{b}} s$ is an outer reduction, then $n f_{\mathrm{w}}$ $(C[]) \equiv C^{\prime}[]$, that is, $C^{\prime}$ contains precisely one hole. Hence, $n f_{\mathrm{w}}(t) \rightarrow_{\mathrm{b}} n f_{\mathrm{w}}(s)$ follows.

THEOREM B.5. Let the TRSs $R_{b}$ and $R_{w}$ be terminating. If $R_{b}$ is noncollapsing and $R_{w}$ is left-linear and confluent, then the direct sum $R_{b} \oplus R_{w}$ is terminating. 
Proof. By induction on $\operatorname{rank}(t)=k$. The basic step $k=1$ is trivial. Induction step: Assume the theorem for $\operatorname{rank}(t)<k$. Then, we will show the case $\operatorname{rank}(t)=k$. Suppose there exists an infinite reduction $t \rightarrow t^{\prime} \rightarrow t^{\prime \prime} \rightarrow \cdots$. From the induction hypothesis, all the terms $t, t^{\prime}, t^{\prime \prime}, \ldots$ have the same rank. In this infinite reduction we must have infinitely many outer reduction steps; otherwise, we must have an infinite internal reduction, in contradiction with the induction hypothesis. Now consider the following cases.

Case 1. root $(t) \in \mathscr{F}_{\mathrm{b}}$. The infinite reduction $t \rightarrow t^{\prime} \rightarrow t^{\prime \prime} \rightarrow \ldots$ has the form

$$
\gamma: t \equiv t_{0} \rightarrow_{\mathrm{b}} t_{1} \rightarrow_{\mathrm{w}} t_{2} \rightarrow_{\mathrm{b}} t_{3} \rightarrow_{\mathrm{w}} t_{4} \rightarrow_{\mathrm{b}} \ldots
$$

Then by Lemma B.4 we have

$$
\gamma^{\prime}: n f_{\mathrm{w}}\left(t_{0}\right) \rightarrow_{\mathrm{b}} n f_{\mathrm{w}}\left(t_{1}\right) \equiv n f_{\mathrm{w}}\left(t_{2}\right) \rightarrow_{\mathrm{b}} n f_{\mathrm{w}}\left(t_{3}\right) \equiv n f_{\mathrm{w}}\left(t_{4}\right) \rightarrow_{\mathrm{b}} \ldots
$$

Since the infinite reduction $\gamma$ has infinitely many steps of outer reduction $\rightarrow_{\mathrm{b}}$, by Lemma B.4 it follows that the reduction $\gamma$ must have infinitely many $\rightarrow_{\mathrm{b}}$ steps: contradiction.

Case 2. $\operatorname{root}(t) \in \mathscr{F}_{\mathrm{w}}$. By using outer reduction steps $\rightarrow_{0}$ and inner reduction steps $\rightarrow_{\mathrm{i}}$, the infinite reduction can be presented as

$$
\delta: t \equiv t_{0} \rightarrow_{\mathrm{i}} s_{0} \rightarrow_{\mathrm{o}} t_{1} \rightarrow_{\mathrm{i}} s_{1} \rightarrow_{\mathrm{o}} t_{2} \rightarrow_{\mathrm{i}} \cdots .
$$

From the noncollapsing property of $R_{\mathrm{b}}$, every inner reduction $t_{n} \rightarrow_{\mathrm{i}} s_{n}$ in $\delta$ does not modify the outermost layer of $t_{n}$, that is, we can write $t_{n} \equiv$ $C_{n} \llbracket t_{1}^{n}, \ldots, t_{m}^{n} \rrbracket$ and $s_{n} \equiv C_{n} \llbracket s_{1}^{n}, \ldots, s_{m}^{n} \rrbracket$ where $t_{i}^{n} \rightarrow s_{i}^{n}(i=1, \ldots, m)$.

Hence, by replacing all the internal terms in $\delta$ with $x$, we obtain

$$
\delta^{\prime}: C_{0}[x, \ldots, x] \rightarrow_{\mathrm{w}} C_{1}[x, \ldots, x] \rightarrow_{\mathrm{w}} C_{2}[x, \ldots, x] \rightarrow_{\mathrm{w}} \cdots
$$

where every $\rightarrow_{\mathrm{w}}$ corresponds to $\rightarrow_{\mathrm{o}}$ in $\delta$. Since $\delta$ has infinitely many steps of $\rightarrow_{\mathrm{w}}$ as $\rightarrow_{\mathrm{o}}, \delta^{\prime}$ must be an infinite reduction: contradiction.

ACKNOWLEDGMENTS. We thank A. Middeldorp as well as some anonymous referees for scrutinizing a previous version and suggesting many improvements. We also thank one of the referees for posing some questions leading us to the result in Appendix B.

\section{REFERENCES}

BACHMAIR, L., AND DERSHOwITZ, N. 1986. Commutation, transformation, and termination. In Lecture Notes in Computer Science, vol. 230. Springer-Verlag, New York, pp. 5-20.

BreazU-Tannen, V., AND Gallier, J. 1989. Polymorphic rewriting conserves algebraic strong normalization and confluence. In Lecture Notes in Computer Science, vol. 372, Springer-Verlag, New York, pp. 137-150.

DERSHOWITZ, N. 1981. Termination of linear rewriting systems. Preliminary version. In Proceedings of the 8th ICALP, S. Even and O. Kariv, eds. Lecture Notes in Computer Science, vol. 115. Springer-Verlag, New York, pp. 448-458.

Dershowitz, N., AND Jouannaud, J.-P. 1990. Rewrite systems. In Formal Models and Semantics. Handbooks of Theoretical Computer Science, vol. B, J. van Leeuwen, ed. Elsevier-The MIT Press, Cambridge, Mass., pp. 243-320.

GANZINGER, H., AND GIEGERICH, R. 1987. A note on termination in combinations of heterogenous term rewriting systems. In Bulletin of the EATCS (European Association for Theoretical Computer Science), vol. 37. (Feb.). Mohien, Paderborn, Germany, pp. 22-28.

HUET, G. 1980. Confluent reductions: Abstract properties and applications to term rewriting systems. J. ACM 27, 4 (Oct.), 797-821. 
Huet, G., AND Oppen, D. C. 1980. Equations and rewrite rules: A survey. In Formal Language Theory: Perspectives and Open Problems, R. Book, ed. Academic Press, London, England, pp. 349-405.

KLOP, J. W. 1992. Term rewriting systems. In Handbook of Logic in Computer Science, vol. 2. S. Abramsky, D. Gabbay, and T. Maibaum, eds. Oxford University Press, Oxford, England, pp. 1-116.

Klop, J. W., Middeldorp, A., TOYama, Y., AND de VRIJer, R. 1994. Modularity of confluence: A simplified proof. Inf. Proc. Lett. 49, 2 (Jan.), 101-109.

KURIHARA, M., AND KAJI, I. 1988/1990. Modular term rewriting systems: Termination, confluence and strategies. Report. Hokkaido Univ., Sapporo, Japan. (Abridged version: Modular term rewriting systems and the termination. Inf. Proc. Lett. 34, 1990, 1-4.)

KURIHARA, M., AND OHUCHI, A. 1989/1990. Modularity of simple termination of term rewrting systems. Rep. 89-SF-31, Hokkaido Univ., Sapporo, Japan. (Also in J. IPS Japan 31, 5, 633-642.)

MIDDELDORP, A. 1989a. A sufficient condition for the termination of term rewriting systems. In Proceedings of the 4th IEEE Symposium on Logic in Computer Science (Asidomar). IEEE, New York, pp. 396-401.

MIDDELDORP, A. 1989b. Modular aspects of properties of term rewriting systems related to normal forms. In Proceedings of the 3rd International Conference on Rewriting Techniques and Applications (Chapel Hill, N.C.). Lecture Notes in Computer Science, vol. 355. Springer-Verlag, New York, pp. 263-277.

Middeldorp, A. 1990. Modular properties of term rewriting systems. Ph.D. dissertation. Free Univ. Amsterdam, Amsterdam, The Netherlands.

Middeldorp, A., AND TOYAMA, Y. 1991. Completeness of combinations of constructor systems. In Proceedings of the 4th International Conference on Rewriting Techniques and Applications (Como). Lecture Notes in Computer Science, vol. 488. Springer-Verlag, New York, pp. 188-199.

OKADA, M. 1989. Strong normalizability for the combined system of the typed lambda calculus and an arbitrary convergent term rewrite system. In Proceedings of the ACM-SIGSAM 1989 International Symposium on Symbolic and Algebraic Computation (Portland, Ore., July 17-19). ACM, New York, pp. 357-363.

Rusinowitch, M. 1987. On termination of the direct sum of term rewriting systems. Inf. Proc. Lett. 26, 65-70.

TOYAMA, Y. 1987a. On the Church-Rosser property for the direct sum of term rewriting systems. J. ACM 34, 1 (Jan.), 128-143.

TOYAMA, Y. 1987b. Counterexamples to termination for the direct sum of term rewriting systems. Inf. Proc. Lett. 25, 141-143.

Toyama, Y. 1988. Commutativity of term rewriting systems. In Programming of Future Generation Computers II, K. Fuchi and L. Kott, eds. North-Holland, Amsterdam, The Netherlands, pp. 393-407.

Toyama, Y., Klop, J. W., AND Barendregt, H. P. 1989. Termination for the direct sum of left-linear term rewriting systems. Preliminary draft. In Proceedings of the 3rd International Conference on Rewriting Techniques and Applications (Chapel Hill, N.C.). Lecture Notes in Computer Science, vol. 355. Springer-Verlag, New York, pp. 477-491.

RECEIVED MAY 1989; REVISED APRIL 1994; ACCEPTED APRIL 1994 